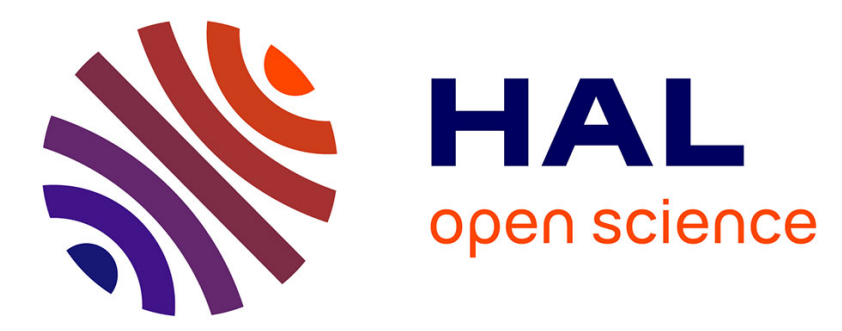

\title{
Solar Wind Dynamic Pressure Upstream From Saturn: Estimation From Magnetosheath Properties and Comparison With SKR
}

\author{
M. Thomsen, C. Jackman, L. Lamy
}

\section{- To cite this version:}

M. Thomsen, C. Jackman, L. Lamy. Solar Wind Dynamic Pressure Upstream From Saturn: Estimation From Magnetosheath Properties and Comparison With SKR. Journal of Geophysical Research Space Physics, 2019, 124 (10), pp.7799-7819. 10.1029/2019JA026819 . hal-03127788

\section{HAL Id: hal-03127788 \\ https://hal.science/hal-03127788}

Submitted on 10 Nov 2021

HAL is a multi-disciplinary open access archive for the deposit and dissemination of scientific research documents, whether they are published or not. The documents may come from teaching and research institutions in France or abroad, or from public or private research centers.
L'archive ouverte pluridisciplinaire HAL, est destinée au dépôt et à la diffusion de documents scientifiques de niveau recherche, publiés ou non, émanant des établissements d'enseignement et de recherche français ou étrangers, des laboratoires publics ou privés.

$$
\text { Copyright }
$$




\section{JGR Space Physics}

\author{
RESEARCH ARTICLE \\ 10.1029/2019JA026819 \\ Key Points: \\ - Analytical development of method \\ to estimate upstream solar wind \\ parameters from Cassini \\ measurements in Saturn's \\ magnetosheath \\ - Results in generally good agreement \\ with solar wind speed and dynamic \\ pressure estimated by other means \\ - Some several-day intervals are found \\ where there is good tracking \\ between the inferred dynamic \\ pressure and SKR intensities
}

Correspondence to:

M. F. Thomsen,

mthomsen@psi.edu

Citation:

Thomsen, M. F., Jackman, C. M., \& Lamy, L. (2019). Solar wind dynamic pressure upstream from Saturn: Estimation from magnetosheath properties and comparison with SKR. Journal of Geophysical Research: Space Physics, 124, 7799-7819. https://doi.org/ 10.1029/2019JA026819

Received 9 APR 2019

Accepted 27 MAY 2019

Accepted article online 7 JUN 2019

Published online 17 OCT 2019

(c)2019. American Geophysical Union. All Rights Reserved.

\section{Solar Wind Dynamic Pressure Upstream From Saturn: Estimation From Magnetosheath Properties and Comparison With SKR}

\author{
M. F. Thomsen ${ }^{1,2}$ (D) C. M. Jackman ${ }^{3}$ (D), and L. Lamy ${ }^{4}$ (D) \\ ${ }^{1}$ Planetary Science Institute, Tucson, AZ, USA, ${ }^{2}$ Los Alamos National Laboratory, Los Alamos, NM, USA, ${ }^{3}$ Department of \\ Physics and Astronomy, University of Southampton, Southampton, UK, ${ }^{4}$ LESIA, Observatoire de Paris, Université PSL, \\ CNRS, Sorbonne Universités, Université Paris Diderot, Meudon, France
}

Abstract An analytical method is developed by which measurements made by the Cassini spacecraft in Saturn's magnetosheath can be used to infer the upstream solar wind parameters, specifically the solar wind speed $\left(V_{\mathrm{sw}}\right)$ and the dynamic pressure $(P d)$. The method is validated by comparing the results with other estimates of these parameters, including the mSWiM MHD model and magnetopause and bow shock models applied to observed boundary crossings. The comparisons suggest that the new inferred $V_{\mathrm{sw}}$ are on average $\sim 40 \mathrm{~km} / \mathrm{s}$ lower than the mSWiM values, and the dynamic pressure values are slightly lower as well. We find few of the lower $P d$ values predicted by mSWiM, probably because Cassini would have been inside the expanded magnetosphere under such conditions. Systematic temporal variations such as interplanetary shocks do seem to be captured well, with arrival times within several days of the MHD prediction. Compared to dynamic pressures estimated from boundary crossings with well-known magnetopause and bow shock models, the magnetosheath-inferred dynamic pressure tends to be somewhat lower, but within the uncertainties of the analytical derivation. Comparison of the inferred dynamic pressure with observed Saturn's kilometric radiation (SKR) activity reveals several episodes of very good temporal tracking between dynamic pressure and SKR intensity, with relatively short time delays (4-5 hr), suggesting rather direct driving. Such good tracking intervals occur almost exclusively on the dawnside of the magnetosphere, where the dominant SKR source is visible. When the tracking is good, the SKR fluxes vary roughly as the square of the dynamic pressure.

Plain Language Summary The extent to which Saturn's magnetosphere may be driven by variations in the upstream solar wind is not well understood, in part because single-spacecraft missions provide no monitor of the upstream plasma to accompany the in situ magnetospheric measurements. One global measure of magnetospheric activity is Saturn's kilometric radiation (SKR). Remote observations of SKR can be combined with solar wind measurements to explore the solar wind's influence on the magnetosphere. We propose a method for greatly increasing the amount of time for which the upstream conditions can be known by using the large database of measurements made by Cassini when it was in Saturn's magnetosheath, the shocked solar wind that coats the sunward side of the magnetosphere. The resulting parameters are validated by comparison with other estimates. Then comparison with Cassini SKR observations reveals that at some times the SKR fluxes clearly track the variations in the solar wind dynamic pressure, suggesting a rather direct solar wind driving of at least some of the magnetospheric processes that produce SKR.

\section{Introduction}

Saturn's magnetosphere is largely internally driven by the planet's rapid rotation, its strong magnetosphereionosphere coupling, and the presence of a vigorous source of plasma deep within the magnetosphere, namely, the water plumes of Enceladus. The dynamical imperative to shed the plasma continuously produced in the inner magnetosphere is accomplished by a sequence of events that are driven by the rapid near-corotation of the magnetospheric plasma: first, outward transport of new pickup plasma through a centrifugally driven flux-tube interchange instability and second, outward centrifugal stretching of those loaded flux tubes until they undergo magnetic reconnection, releasing the picked-up plasma in the form of largescale plasmoids or possibly smaller scale drizzle (e.g., Blanc et al., 2015; Krupp et al., 2018; Thomsen, 2013). The magnetospheric dynamics at Saturn are thus quite different from those at the Earth, where the 
primary driver is the interaction between the solar wind and the magnetosphere, particularly via dayside and nightside magnetic reconnection.

Nonetheless, there is abundant evidence that the solar wind does in fact affect Saturn's magnetospheric dynamics. At the most basic level, the size of the magnetosphere responds to the solar wind dynamic pressure (e.g., Arridge et al., 2006; Kanani et al., 2010; Pilkington et al., 2015). Moreover, the disk-like structure that arises from centrifugal stretching of the magnetic field is more prominent at low solar wind dynamic pressure, and the hinging distance of the plasma sheet disk varies with dynamic pressure as well (Arridge et al., 2008).

Dayside magnetopause reconnection, which is the primary driver of terrestrial magnetospheric dynamics, is also known to occur at Saturn, based both on in situ evidence (e.g., Badman et al., 2013; Fuselier et al., 2014; Huddleston et al., 1997; Jasinski et al., 2014; Lai et al., 2012; Masters et al., 2012; McAndrews et al., 2008) and on remote observations of the aurora and polar cap (e.g., Badman et al., 2005, 2014; Belenkaya et al., 2008, 2011; Lamy et al., 2018; Radioti et al., 2011). While the reconnection voltage that can be developed at Saturn is rarely large enough to compete with corotation for driving magnetospheric dynamics (e.g., Badman \& Cowley, 2007; Masters et al., 2014), the loading of opened flux into the magnetospheric tail over a longer time interval can lead to episodes of significant tail reconnection and plasmoid release (e.g., Badman et al., 2014; Bunce et al., 2005; Jackman et al., 2011). Such episodes are attributed to sudden increases in the solar wind dynamic pressure (e.g., Bunce et al., 2005; Cowley et al., 2005; Jackman et al., 2005, 2010; Jia et al., 2012; Kidder et al., 2012; Zieger et al., 2010). Moreover, there is evidence that under the influence of prolonged elevated dynamic pressure, which is typical of recurrent solar wind corotating interaction regions, the tail plasma sheet can erode away until open lobe field lines are reconnecting, initiating an Earth-like Dungey dynamic cycle (Thomsen et al., 2015).

One prominent indicator of solar wind influence on Saturn's magnetospheric activity is the association between increases in the solar wind dynamic pressure and the flux of Saturn kilometric radiation (SKR) emitted above the magnetic poles (Badman et al., 2008; Desch, 1982; Desch \& Rucker, 1983, 1985; Jackman et al., 2005, 2010; Kimura et al., 2013; Kurth et al., 2005; Lamy, 2017; Taubenschuss et al., 2006). There is a similar association between increases in the dynamic pressure and enhanced auroral emissions (e.g., Clarke et al., 2005, 2009; Crary et al., 2005; Lamy, 2017). The link between SKR enhancements, auroral emissions, and magnetotail activity (e.g., Jackman et al., 2009, 2010; Kurth et al., 2016; Mitchell et al., 2005; Reed et al., 2018) completes the circle connecting dynamic pressure enhancements with tail dynamics and SKR activity (see also Bunce et al., 2010).

The difficulty with assessing the degree of solar wind influence on Saturn's magnetospheric dynamics with a single-spacecraft mission like Cassini is that, lacking an upstream monitor, one cannot know the actual solar wind conditions when Cassini is in the magnetosphere. On the time scale of one to a few days, Roussos et al. (2017) have shown that Cassini MIMI/LEMMS observations of solar energetic particles and galactic cosmic rays, both of which penetrate well inside the magnetosphere, can put magnetospheric observations into the context of the global structure of the solar wind (specifically, coronal mass ejections and corotating interaction regions). However, on a finer time scale, most associations of magnetospheric activity with solar wind driving depend on remote observations like auroral ultraviolet emissions and SKR, as described above, while Cassini itself is within the upstream solar wind. A further difficulty is that there are actually rather few useful solar wind intervals in the Cassini data because of the frequently unfavorable viewing of the Cassini Plasma Spectrometer (CAPS), which provides solar wind measurements (e.g., Crary et al., 2005). This is because CAPS does not face into the solar wind when the Cassini imaging instruments are viewing the planet and its vicinity.

Several studies have sought to quantify the association between $P d$ (the solar wind dynamic pressure) and SKR fluxes. Desch and Rucker $(1983,1985)$ used superposed epoch analyses of Voyager data to determine that $P d\left(=\rho V^{2}\right.$, where $\rho$ is the solar wind mass density and $\mathrm{V}$ is the flow speed $)$ and related quantities $\left(\rho \mathrm{V}\right.$ and $\left.\rho \mathrm{V}^{3}\right)$ are significant SKR drivers, with the highest correlation coefficients at zero time lag (using a time resolution of $10.66 \mathrm{hr}$ ). Desch (1983) argued further that the disappearance of the solar wind stress imposed on Saturn's magnetosphere (when the planet was transiently immersed in Jupiter's magnetotail during the Voyager 2 approach) yields a complete disappearance of SKR signal. 
In a refinement of the pioneering work of Desch \& Rucker, Taubenschuss et al. (2006) used linear prediction analyses on 17 days of post-SOI Cassini data to reanalyze the solar wind influence. They explored 10 different parameters involving solar wind density, flow speed, temperature, and magnetic field strength and orientation. There were two clear SKR enhancements within the data set they used, which was obtained at $\sim 150 R_{S}$ from the planet near dawn local time. They found that variations in solar wind density are a stronger driver than variations in the solar wind speed. Using a low-pass filter ( $5 \mathrm{hr}$ ) on 20-min SKR data, they found fairly long lag times between the solar wind parameters and the presumed SKR response: the best prediction was found with $P d$, with a time delay of $\sim 13 \mathrm{hr}$ from arrival at the nose of the magnetopause.

Similarly, Badman et al. (2008) used Cassini data obtained in the solar wind to examine the relationship between solar wind structure and SKR enhancements. Using primarily the measured solar wind magnetic field strength, they identified a number of intervals where field enhancements (and presumably corresponding dynamic pressure enhancements) were clearly associated with SKR enhancements. In the one event where they had solar wind plasma data to accompany the field data, they found that forward interplanetary shocks led to increased SKR intensities, and a reverse shock, where the dynamic pressure and magnetic field strength declined, was associated with a clear decrease in SKR that lasted approximately two days. They also sought to quantify the time delay between the solar wind structures and the corresponding SKR signatures, but for a number of their events the uncertainties in the propagation time from Cassini's location to the planet were too large to make useful timing assessments. In the case of three events where the propagation delays were presumably quite low (when Cassini was near the local dawn), they found a large variation in the apparent delay between the solar wind and the SKR, from as short as 1 to $10 \mathrm{hr}$.

Lamy et al. (2018) compared SKR activity with model-predicted solar wind properties for the first 260 days of 2017. They found that the strongest enhancements of SKR were associated with predicted Pd enhancements. They concluded that solar wind and planetary rotation both control SKR activity and UV aurorae: solarwind-induced magnetospheric compressions trigger long-lasting (more than a planetary rotation) global SKR enhancements that extend toward low frequencies (Bunce et al., 2010; Desch, 1982; Kurth et al., 2005, 2016; Lamy et al., 2010), while shorter SKR intensifications are associated with rotationally modulated nightside injections (Jackman et al., 2009; Lamy et al., 2013; Mitchell et al., 2009; Reed et al., 2018). Similar conclusions were reached by Reed et al. (2018) based on observations of low-frequency extensions of SKR power spectra.

One major complication in assessing the influence of solar wind properties on magnetospheric dynamics by using SKR as a proxy for magnetospheric activity is that SKR is beamed anisotropically, so the detection of SKR activity is dependent on the observer's position (see the review by Lamy, 2017, and references therein). In particular, SKR is best observed when Cassini is near the equatorial plane and in the dawn local time sector (Kimura et al., 2013; Lamy et al., 2008). This visibility condition will be a factor in the observations we present below.

Recently, we have provided a complete listing of intervals when Cassini was in the magnetosheath (the region of shocked solar wind between the bow shock and the magnetopause) and during which the field of view of the CAPS Ion Mass Spectrometer contained the transonic magnetosheath ion distribution, so that reliable ion moments (density, temperature, and flow velocity) could be derived (Thomsen et al., 2018). Using those measurements, an empirical relationship between the observed energy per magnetosheath particle and the upstream solar wind speed was established. The ability to use magnetosheath observations to infer upstream solar wind properties greatly enlarges the upstream data set available for correlation studies with remote sensing measurements, as described above. In the present paper, this capability is significantly expanded and placed on a firm analytic foundation. We derive here an analytical expression that allows us to use Cassini magnetosheath measurements to infer the upstream dynamic pressure, not just the flow speed. The derivation of the analytical expression also yields an estimate of the upstream flow speed that significantly improves on the empirical relationship obtained earlier (Thomsen et al., 2018). The large set of upstream parameters estimated in this way is then used to explore the SKR response to variable solar wind.

\section{Data}

The principal data used in this study are the magnetosheath parameters provided as supporting information in our earlier paper (Thomsen et al., 2018). That data set consists of the numerical ion moments from 
CAPS/Ion Mass Spectrometer (proton density, temperature, and flow velocity, and alpha particle density and temperature), the corresponding time averages of the electron moments (density and temperature) from the CAPS Electron Spectrometer, the energetic particle pressures from MIMI, and the magnetic field measurements from the Cassini magnetometer (MAG). The data set includes 657 separate magnetosheath intervals, comprising a total of 19,155 valid measurements (2,213 hr). Each entry in the file is based on 416 s of data.

The upstream parameters inferred from the magnetosheath data are compared to 1-hr values predicted by the mSWiM MHD solar wind model, which propagates 1 A.U. solar wind observations to Saturn's orbit (Zieger \& Hansen, 2008). Inferred upstream dynamic pressures are also compared with the solar wind dynamic pressures estimated from the set of magnetopause and bow shock crossings identified by Jackman et al. (2019). As in that study, $P d$ is calculated from observed magnetopause crossings using the model of Kanani et al. (2010), and from observed bow shock crossings using the model of Went et al. (2011).

Finally, our inferred upstream parameters are compared with SKR activity quasi-continuously measured by the Cassini Radio and Plasma Wave Investigation (RPWS; Gurnett et al., 2004). Specifically, we use 3-min averages of the SKR flux densities between 100 and $300 \mathrm{kHz}$ (Lamy et al., 2008).

\section{Relationship of Magnetosheath Parameters to Solar Wind Dynamic Pressure: Theory}

In our earlier manuscript, we provided an ad hoc empirical relationship between the energy per particle within the magnetosheath and the upstream solar wind speed. Here we improve on that relationship by developing a first-principle derivation of an analytical relationship between measureable magnetosheath parameters and upstream conditions. We begin with the Rankine-Hugoniot jump conditions for a onedimensional boundary with zero width, assuming ideal MHD (zero resistivity):

$$
\begin{gathered}
\widehat{\boldsymbol{n}} \cdot[\rho \boldsymbol{u}]=0 \\
\widehat{\boldsymbol{n}} \cdot\left[\left(\frac{1}{2} u^{2}+\frac{\gamma p}{(\gamma-1) \rho}+\frac{B^{2}}{\mu_{0} \rho}\right) \rho \boldsymbol{u}\right]-\frac{1}{\mu_{0}} \widehat{\boldsymbol{n}} \cdot[(\boldsymbol{u} \cdot \boldsymbol{B}) \boldsymbol{B}]=0
\end{gathered}
$$

where $[. .$.$] represents the jump in the parameters across the boundary. In these equations, \widehat{\boldsymbol{n}}$ is the normal to the boundary, $\rho$ is the plasma mass density, $\mathbf{u}$ is the flow velocity of the plasma, $B$ is the magnetic field strength, and $\gamma$ is the polytropic index, which we take to be $5 / 3$.

If we take $\widehat{\boldsymbol{n}}=\widehat{\boldsymbol{u}}$, where $\widehat{\boldsymbol{u}}=\boldsymbol{u} / u$, then we find that along a magnetosheath streamline, equation (2) gives

$$
\left(\frac{1}{2} u^{2}+\frac{\gamma}{(\gamma-1) \rho}+\frac{B^{2}}{\mu_{0} \rho}\right) \rho u-\frac{u}{\mu_{0}}(\widehat{\boldsymbol{u}} \cdot \boldsymbol{B})^{2}=\mathrm{const}
$$

Combining the two terms, with $\widehat{\boldsymbol{u}} \cdot \widehat{\boldsymbol{b}}=\cos \theta_{U B}$,

$$
u\left(\frac{1}{2} \rho u^{2}+\frac{\gamma p}{(\gamma-1)}+\frac{B^{2}}{\mu_{0}} \sin ^{2} \theta_{U B}\right)=\text { const }
$$

Because the plasma beta and the Alfvén Mach number in the magnetosheath flow are typically well above 1 (Thomsen et al., 2018), we can neglect the magnetic part of equation (3), yielding

$$
u\left(\frac{1}{2} \rho u^{2}+\frac{\gamma p}{(\gamma-1)}\right) \approx \text { const }
$$

Similarly, if we take equation (2) with $\widehat{\boldsymbol{n}}=\widehat{\boldsymbol{n}}_{B S}$, the bow shock normal at a given location, we get the shock jump equation 


$$
\left(\frac{1}{2} u_{1}^{2}+\frac{\gamma p_{1}}{(\gamma-1) \rho_{1}}\right) \rho_{1} u_{1} \cos \theta_{U n 1} \approx\left(\frac{1}{2} u_{2}^{2}+\frac{\gamma p_{2}}{(\gamma-1) \rho_{2}}\right) \rho_{2} u_{2} \cos \theta_{U n 2}
$$

where $\theta_{U n 1}$ is the angle between the upstream (solar wind) flow velocity and the bow shock normal, and $\theta_{U n 2}$ is the same for the magnetosheath flow immediately downstream of the shock. Again, because the Alfvén Mach number is generally well above 1, we have dropped the magnetic field terms.

From the MHD continuity equation (equation (1) with $\widehat{\boldsymbol{n}}=\widehat{\boldsymbol{n}}_{B S}$ ), the mass flow normal to the shock is conserved:

$$
\rho_{1} u_{1} \cos \theta_{U n 1}=\rho_{2} u_{2} \cos \theta_{U n 2}
$$

so equation (5) becomes

$$
\left(\frac{1}{2} u_{1}^{2}+\frac{\gamma p_{1}}{(\gamma-1) \rho_{1}}\right) \approx\left(\frac{1}{2} u_{2}^{2}+\frac{\gamma p_{2}}{(\gamma-1) \rho_{2}}\right)
$$

In the upstream solar wind, the sonic Mach number is very high, so we can further neglect the thermal contribution to the left-hand side, yielding

$$
\begin{aligned}
\frac{1}{2} u_{1}^{2} & \approx\left(\frac{1}{2} u_{2}^{2}+\frac{\gamma p_{2}}{(\gamma-1) \rho_{2}}\right) \\
& \approx\left(\frac{1}{\rho_{2} u_{2}}\right) u_{2}\left(\frac{1}{2} \rho_{2} u_{2}^{2}+\frac{\gamma p_{2}}{(\gamma-1)}\right) \\
& \approx\left(\frac{1}{\rho_{2} u_{2}}\right) u\left(\frac{1}{2} \rho u^{2}+\frac{\gamma p}{(\gamma-1)}\right)
\end{aligned}
$$

where we have used equation (4) in the final step. Equation (7) now relates the upstream flow speed to the downstream magnetosheath parameters directly behind the shock (subscript 2) and along that streamline throughout the magnetosheath (unsubscripted).

Finally, using the continuity equation (1), again with $\widehat{\mathbf{n}}=\widehat{\mathbf{u}}$, we get $\rho u=$ constant along a streamline, so

$$
\frac{1}{2} u_{1}^{2} \approx\left(\frac{1}{2} u^{2}+\frac{\gamma p}{(\gamma-1) \rho}\right)
$$

i.e.,

$$
\begin{aligned}
u_{1} & \approx \sqrt{2\left(\frac{1}{2} u^{2}+\frac{\gamma p}{(\gamma-1) \rho}\right)} \\
& \approx u \sqrt{1+5 \frac{p}{\rho u^{2}}}
\end{aligned}
$$

which now relates the upstream flow speed to magnetosheath parameters at any point in the downstream region. Equation (8) roughly validates our previous argument (Thomsen et al., 2018) that $u_{1}$ can be empirically approximated by

$$
\frac{1}{2} m_{p} u_{1}^{2} \approx 1.860 \cdot\left(k T_{p}+\frac{1}{2} m_{p} u^{2}\right)
$$

i.e.,

$$
u_{1} \approx \sqrt{2 \cdot 1.860 \cdot\left(\frac{k T_{p}}{m_{p}}+\frac{1}{2} u^{2}\right)} \approx 1.36 u \sqrt{1+\frac{2 p_{p}}{\rho_{p} u^{2}}}
$$

where $p_{p}$ and $\rho_{p}$ indicate the proton portion of the pressure and temperature. Equation (8) actually suggests that the earlier study might have been better fit using the multiplicative term $\gamma /(\gamma-1)$ to weight the thermal pressure, which may have eliminated the need for the arbitrary normalization constant 1.36 . 


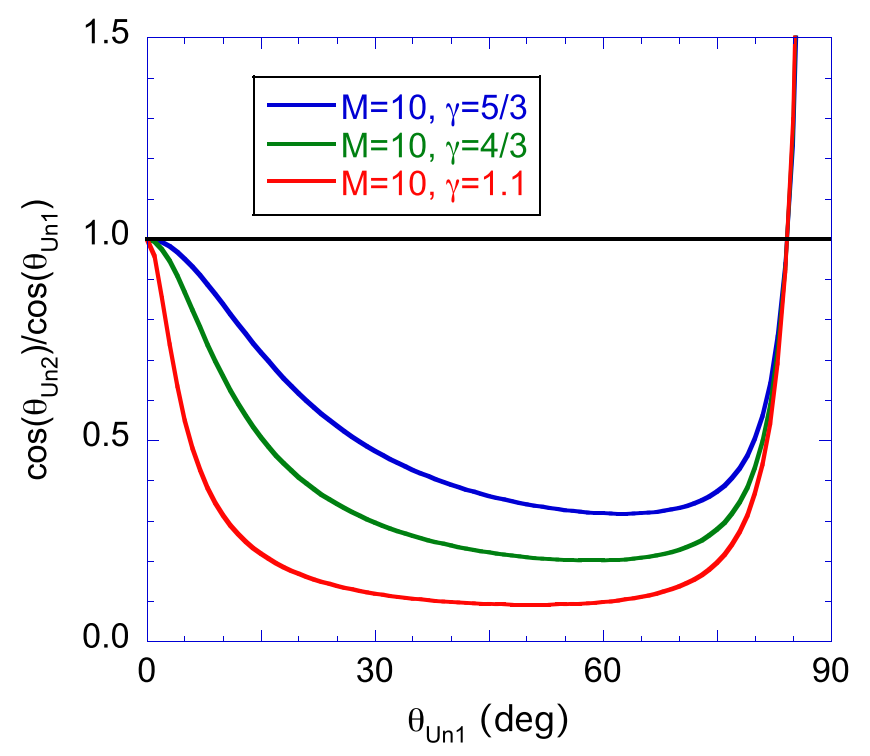

Figure 1. Ratio of $\cos \theta_{U n 2}$ to $\cos \theta_{U n 1}$ as a function of $\theta_{U n 1}$ for Mach numbers $\mathrm{M}=5,10,20$.
If we multiply equation (7) by $\rho 1$, we get

$$
\begin{aligned}
\frac{1}{2} \rho_{1} u_{1}^{2} \approx & \left(\frac{\rho_{1}}{\rho_{2}}\right)\left(\frac{u}{u_{2}}\right)\left(\frac{1}{2} \rho u^{2}+\frac{\gamma p}{(\gamma-1)}\right) \\
& \approx\left(\frac{\rho_{1}}{\rho_{2}}\right)\left(\frac{u_{1}}{u_{2}}\right)\left(\frac{u}{u_{1}}\right)\left(\frac{1}{2} \rho u^{2}+\frac{\gamma p}{(\gamma-1)}\right) \\
& \approx\left(\frac{\cos \theta_{U n 2}}{\cos \theta_{U n 1}}\right)\left(\frac{u}{u_{1}}\right)\left(\frac{1}{2} \rho u^{2}+\frac{\gamma p}{(\gamma-1)}\right)
\end{aligned}
$$

where we have again used the mass conservation equation to relate $\rho_{1} u_{1}$ to $\rho_{2} u_{2}$. Using equation (8) for $u_{1}$ in the denominator of the RHS:

$$
\begin{aligned}
\rho_{1} u_{1}^{2} \approx & \approx\left(\frac{\cos \theta_{U n 2}}{\cos \theta_{U n 1}}\right) 2 \rho u \frac{\left(\frac{1}{2} u^{2}+\frac{\gamma p}{(\gamma-1) \rho}\right)}{\sqrt{2\left(\frac{1}{2} u^{2}+\frac{\gamma p}{(\gamma-1) \rho}\right)}} \\
& \approx\left(\frac{\cos \theta_{U n 2}}{\cos \theta_{U n 1}}\right) 2 \rho u \sqrt{\frac{1}{2}\left(\frac{1}{2} u^{2}+\frac{\gamma p}{(\gamma-1) \rho}\right)} \\
& \approx\left(\frac{\cos \theta_{U n 2}}{\cos \theta_{U n 1}}\right) \rho u^{2} \sqrt{\left(1+\frac{2 \gamma p}{(\gamma-1) \rho u^{2}}\right)} \\
& \approx\left(\frac{\cos \theta_{U n 2}}{\cos \theta_{U n 1}}\right) \rho u^{2} \sqrt{\left(1+5 \frac{p}{\rho u^{2}}\right)}
\end{aligned}
$$

The first term on the RHS of equation (10) is the ratio of the cosines of the angles between the upstream/downstream flow directions and the shock normal at the point where the observed streamline originally crossed the shock. A fast shock bends the flow away from the normal direction, so this term will always be less than or equal to 1 . Within the hydrodynamic flow approximation we have used (by neglecting the magnetic field terms in all the equations), there are a number of formulations that relate $\theta_{U n 2}$ and $\theta_{U n 1}$, based on shock jump equations. Using the formulation of Rathakrishnan (2010), equation 4.13) gives

$$
\tan \left(\theta_{U n 2}-\theta_{U n 1}\right)=2 \tan \theta_{U n 1}\left[\frac{M^{2} \cos ^{2} \theta_{U n 1}-1}{M^{2}\left(\gamma-\cos 2 \theta_{U n 1}\right)+2}\right]
$$

Solving for $\theta_{U n 2}$ as a function of $\theta_{U n 1}$, we find

$$
\theta_{U n 2}=\theta_{U n 1}+\tan ^{-1}\left\{2 \tan \theta_{U n 1}\left[\frac{M^{2} \cos ^{2} \theta_{U n 1}-1}{M^{2}\left(\gamma-\cos 2 \theta_{U n 1}\right)+2}\right]\right\}
$$

Figure 1 shows the resulting ratio of cosines for $M=5,10,20$. Each curve starts at 1 for normal incidence at the nose and declines to a minimum between $\sim 0.3$ and 0.4 , before rising back to 1 at the Mach angle of the shock, $\cos ^{-1}(1 / \mathrm{M})$.

The remaining link in the chain relating the upstream dynamic pressure to the observable magnetosheath parameters is then estimation of the angle $\theta_{U n 1}$ between the upstream flow and the normal to the shock at the point the local streamline crossed the shock. For the Went et al. (2011) bow shock model, the radial distance from Saturn to a point on the shock is

$$
r=\frac{(1+\varepsilon) c_{1} P_{d}^{-1 / c_{2}}}{1+\varepsilon \cos \zeta}
$$

where $\left(\varepsilon, c_{1}\right)=(0.84,15)$ (Went et al., 2011), and $\zeta=\cos ^{-1}(\widehat{\boldsymbol{x}} \cdot \widehat{\boldsymbol{r}})$ is the cylindrical polar angle relative to the solar direction. For this shock shape, the shock normal in the equatorial plane can be shown to depend on $\zeta$ as 


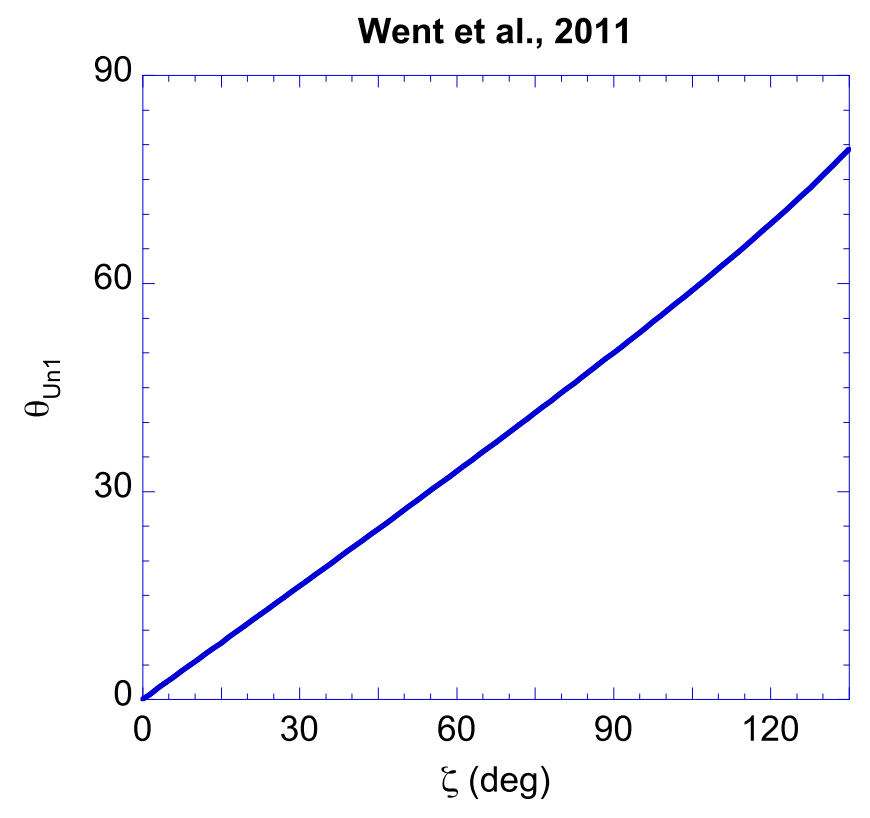

Figure 2. Angle between the upstream flow and the shock normal as a function of $\zeta$ for the Went et al. (2011) model bow shock. $\zeta$ is the angle between the solar direction and a vector from Saturn to a point on the shock.

$$
\widehat{\boldsymbol{n}}=[(\varepsilon+\cos \zeta) \widehat{\boldsymbol{x}}-\sin \zeta \widehat{\boldsymbol{y}}] /\left(1+2 \varepsilon \cos \zeta+\varepsilon^{2}\right)^{1 / 2}
$$

independent of the dynamic pressure and the model parameters other than $\varepsilon$. Thus, the angle between the upstream flow (in the $-\widehat{\boldsymbol{x}}$ direction) and the shock normal is

$$
\theta_{U n 1}=\cos ^{-1}(\widehat{\boldsymbol{x}} \cdot \widehat{\boldsymbol{n}})=\cos ^{-1}\left[\frac{\varepsilon+\cos \zeta}{\left(1+2 \varepsilon \cos \zeta+\varepsilon^{2}\right)^{1 / 2}}\right]
$$

Figure 2 shows the resulting $\theta_{U n 1}$ as a function of $\zeta$ for $\varepsilon=0.84$ (Went et al., 2011).

Of course, it is not possible to know the location $\zeta$ at which the local streamline crossed the shock unless we are very near a bow shock crossing. In general, $\zeta$ is less than this local value. Indeed, when the spacecraft is very near the magnetopause, the local streamlines crossed the shock near the nose $(\zeta=0)$. To estimate the ratio of cosines in equation (10) for any given magnetosheath observation, we therefore take $\zeta$ as the value at the location halfway between the observation point $\zeta_{\text {obs }}$ and the nose of the magnetosphere $\zeta=0$ :

$$
\zeta=\zeta_{\mathrm{obs}} / 2
$$

Finally, to sum up, using the observation location, we use equation (16) to estimate the location at which the local streamline crossed the shock. With equation (15) this gives the shock normal angle for the Went et al. (2011) bow shock. Equation (12) then is used to estimate the angle between the downstream flow and the shock normal. This depends on the Mach number M, but at the relatively high $M$ at Saturn, it is not very sensitive to that parameter, and we simply take $M=10$. With $\theta_{U n 1}$ and $\theta_{U n 2}$ we can then calculate the ratio of the cosines and use the observed dynamic pressure and thermal pressure in equation (10) to deduce the upstream dynamic pressure.

One issue with using the above analysis to relate magnetosheath parameters to upstream parameters is what to take for the magnetosheath pressure, $p$. The difficulty is the frequent presence of suprathermal ions (often $\mathrm{O}+$ ) that leak from the magnetosphere into the magnetosheath (Sergis et al., 2013). These are not really accounted for in the MHD conservation equations, but when they are present, they do contribute significantly to the total particle pressure. For the present analysis, we have performed the calculation both with and without the pressure contributions from the energetic ions and will discuss the results below.

In addition to questions about the energetic-particle contributions to the pressure, the uncertainties in the above determination arise primarily from neglect of the magnetic terms in the energy and momentum conservation equations and in the approximation of the point of transit of the shock for the local streamline. The latter affects the ratio $\cos \theta_{U n 2} / \cos \theta_{U n 1}$, which is probably not wrong by more than a factor of 2 , as shown in Figure 1.

\section{Results}

\subsection{Should Energetic Particles Be Included in the Magnetosheath Pressure?}

Using the procedure described in section 3, the upstream solar wind speed $V=u_{1}$ is estimated using equation (8), and the dynamic pressure $P d=\rho_{1} u_{1}^{2}$ is estimated using equation (10) for all of the points in the magnetosheath data set of Thomsen et al. (2018). As mentioned above, it is not clear whether or not it is necessary to include the contribution of the superthermal ions in the pressure terms in these equations. The red and light blue curves in Figure 3a show the occurrence distributions of the inferred solar wind speed when the total particle pressure or only the plasma pressure, respectively, is used in equation (8). It is clear that the contribution of the energetic particles creates a long high- $\mathrm{V}$ tail on the distribution because of the many instances when the superthermal ion pressure is comparable to or exceeds that in the thermal plasma. For comparison, the green curve in Figure $3 \mathrm{a}$ is the occurrence distribution of solar wind V predicted by the 

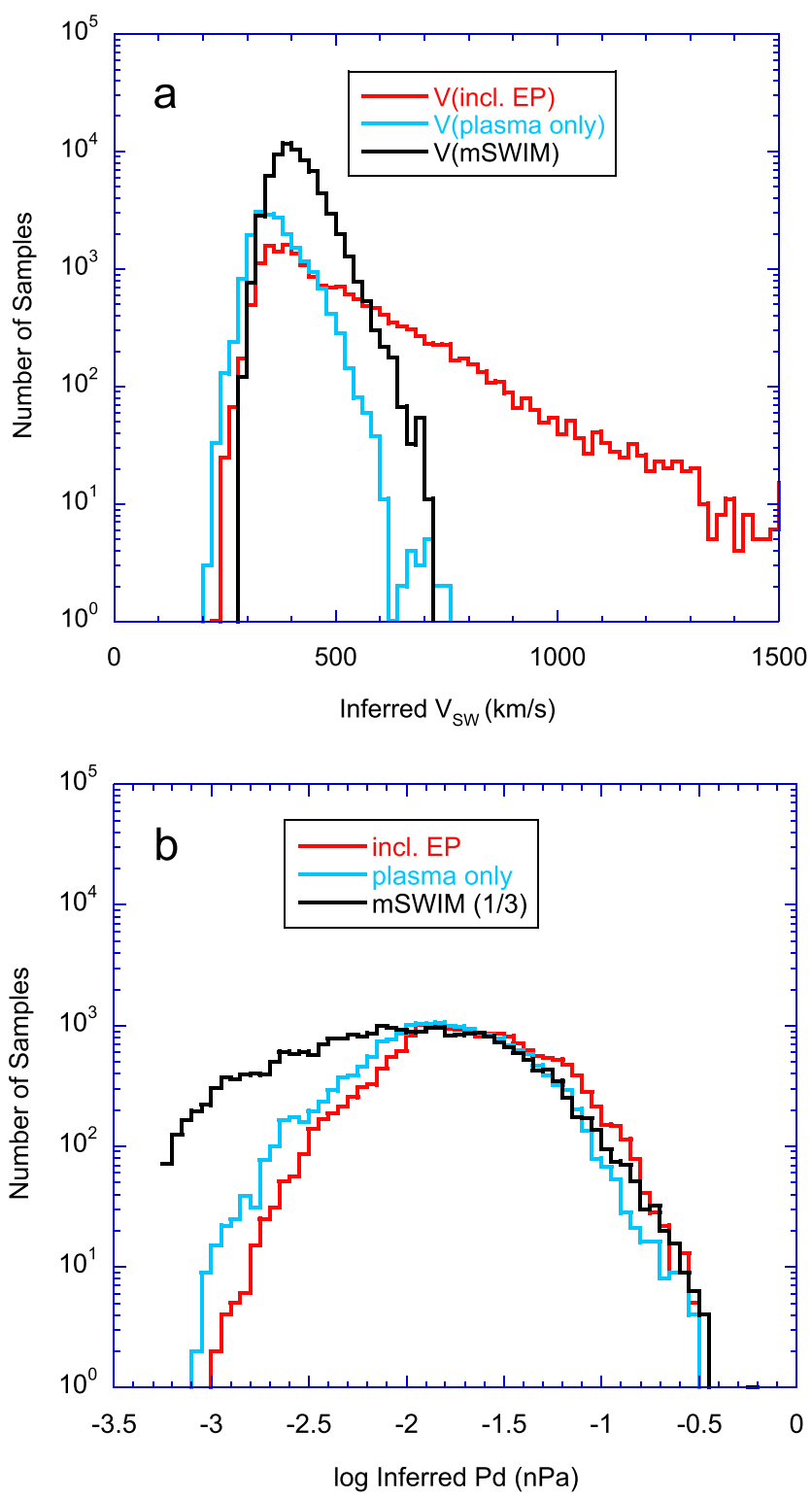

Figure 3. Occurrence distributions of (a) solar wind speed and (b) dynamic pressure calculated from equations (8) and (10), respectively, using the total particle pressure (red curves) and using only the plasma contribution to the pressure (light blue curves). The green curves show the corresponding distributions for the 1-hr solar wind speed and dynamic pressure predicted by the mSWiM MHD model for the time period covered by the magnetosheath measurements (with the number of samples scaled down by a factor of 4 for the $V_{\mathrm{sw}}$ distribution and 3 for the $P d$ distribution). In (b) the $P d$ distributions are overlaid on the distribution from Figure $5 \mathrm{~b}$ of Jackman and Arridge (2011, black curve).
mSWiM MHD model for the interval from mid-2004 to mid-2012, which covers the time range of the magnetosheath data set. The mSWiM distribution is quite similar to the distribution of $\mathrm{V}$ inferred using only the thermal plasma pressure, although there is a small offset that will be further discussed below. The high- $\mathrm{V}$ tail produced by including the superthermal pressure in the calculation (red curve) is absent in the mSWiM predictions.

Figure $3 \mathrm{~b}$ shows similar occurrence distributions for the solar wind dynamic pressure, again as inferred by including the superthermal contribution to the magnetosheath pressure (red curve), by including only the plasma contribution (light blue curve), and as predicted by the mSWiM model over the same time interval (green curve). These distributions are overlaid on Figure 5b from Jackman and Arridge (2011, black curve), who compiled solar wind measurements from the Pioneer 11, Voyager 1, and Voyager 2 flybys of Saturn, and the early part of Cassini's nearSaturn mission. As in the distributions for V, inclusion of the superthermal contribution to the magnetosheath pressure creates a slight shift toward higher values, but there is no pronounced high- $P d$ tail, possibly because the binning has been done logarithmically. At the high-Pd end of the distribution, it is difficult to distinguish any particular discrepancy between either curve and the mSWiM prediction or the Jackman and Arridge findings (we will return to the obvious mSWiM discrepancy at low $P d$ in a subsequent discussion). However, on the basis of the clear high-V discrepancy seen in Figure 3a, we conclude that the energetic particle contribution to the total pressure should not be included in either equation (8) or equation (10).

\subsection{Comparison With $\mathrm{mSWiM} \mathrm{V,Pd}$}

As presented above, Figure 3 compares the solar wind speed and dynamic pressure inferred from magnetosheath measurements with $\mathrm{mSWiM}$ predictions and with earlier solar wind data compiled by Jackman and Arridge (2011). Using only the plasma contribution to the magnetosheath pressure yields distributions that are quite similar to both the model and the previous observations, except for the prominent low-Pd extension of the mSWiM values. There is a slight offset of the magnetosheath-derived $V_{\text {sw }}$ to lower values than mSWiM predicts; indeed, a plot of the probability occurrence distribution of the inferred $V_{\mathrm{sw}}+40 \mathrm{~km} / \mathrm{s}$ is found to overlay perfectly the distribution from mSWiM, except for the bottom $\sim 1 \%$ and top $0.1 \%$ of values (not shown). Thus, on average the inferred $V_{\text {sw }}$ values are $\sim 10 \%$ low compared to mSWiM. The magnetosheath-inferred $P d$ values are somewhat low compared to the Jackman and Arridge values, by $\sim 30 \%$, but they match very well the higher-Pd portion of the mSWiM curve.

Figure 4 presents the time history of the full set of solar wind speeds (Figure 4a) and solar wind dynamic pressures (Figure $4 \mathrm{~b}$ ) inferred from magnetosheath data using equations (8) and (10). The inferred values are plotted as red symbols, and they are compared with the values predicted by the mSWiM MHD model (blue symbols). Since the mSWiM results are based on propagating near-Earth solar wind measurements to Saturn, the prediction should be best when Saturn and Earth are in conjunction and viewing the same solar wind plasma as it propagates outward through the heliosphere. Black bars below the top axis in Figure 4 indicate intervals when the mSWiM parameter $|\mathrm{d} \phi|<75^{\circ}$, where $\mathrm{d} \phi$ is the heliocentric longitudinal separation between Saturn and the Earth. Zieger and Hansen (2008) found broad maxima in the mSWiM prediction efficiency over roughly this range of $d \phi$. 

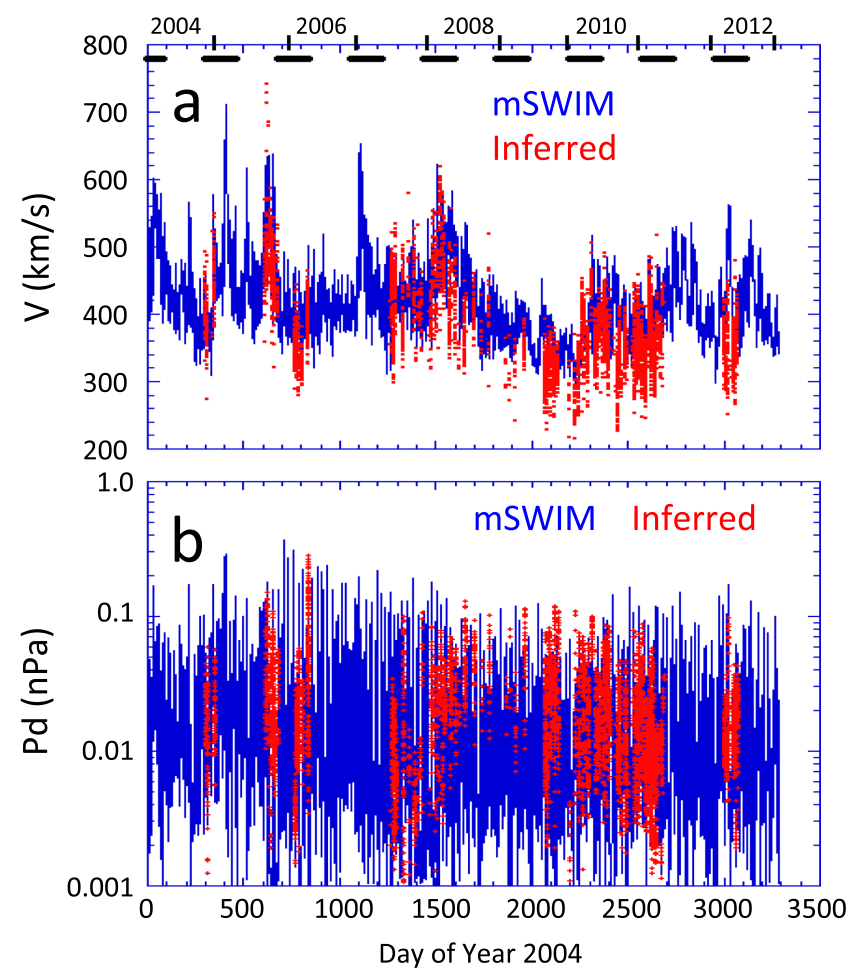

Figure 4. Time history of solar wind speed (Figure 4a) and dynamic pressure (Figure $4 \mathrm{~b}$ ) inferred from magnetosheath measurements according to equations (8) and (10) (red symbols) and as predicted by the mSWiM MHD model (Zieger \& Hansen, 2008; blue curves). Values are plotted as a function of days from 1 January 2004. Black bars below top axis show intervals when $\mathrm{mSWiM}|\mathrm{d} \varphi|<75^{\circ}$, so prediction accuracy should be higher (see text for details).
On this very compressed time scale, Figure 4a shows that the inferred solar wind speed tracks the mSWiM prediction quite well but with a tendency to slightly underestimate the mSWiM value (see also Figure 3a). As shown in Figure 4b, the dynamic pressure is highly variable in time, both in the mSWiM model and as inferred from magnetosheath measurements, primarily because of the large variability in the solar wind density. The range of inferred values does seem to track reasonably well the range of values predicted by mSWiM (e.g., the general decline around day 1,500). And as noted above, the statistical distributions of values are similar, except for the relative absence of low inferred $P d$ values compared to mSWiM.

Figure 5 shows the same quantities as Figure 4, for the first three months of 2008. With some scatter, the inferred values do generally track the $\mathrm{mSWiM}$ predictions, at times with remarkable fidelity. During these three months the heliocentric angle separating Earth and Saturn was less than $50^{\circ}$, with opposition near day 1,516 . In this configuration the mSWiM predictions should be the most accurate (Zieger \& Hansen, 2008). The apparent spread in the inferred $\mathrm{V}$ values in Figure $5 \mathrm{a}$ is due to a combination of variability in the derived magnetosheath flow speed and temperature. Whether this is due to true variability in the upstream medium, or due to waves in the magnetosheath, or whether it is just uncertainty in the measurement is open to further investigation, but it typically results in a variation in the inferred flow speed of $\sim \pm 10 \%$ relative to the mean over the course of a few hours. Similarly, the variability in the inferred $P d$ is due to variability in the density, flow speed, and temperature of the magnetosheath plasma, but close examination of contiguous sequences of magnetosheath measurements suggests that much of that variability is systematic (i.e., real temporal changes), with perhaps $\pm 30 \%$ variations from individual measurement to measurement.

While the temporal tracking of the inferred and predicted values is reasonably good, Figure 5 shows that the times of some of the observed changes in inferred values disagree somewhat with the mSWiM predictions, consistent with the known uncertainties in arrival times discussed extensively by Zieger and Hansen (2008). It is also noteworthy that the magnetosheath-inferred dynamic pressure rarely extends much below $0.01 \mathrm{nPa}$, thus missing the very low values predicted by $\mathrm{mSWiM}$ in the rarefaction regions behind the apparent stream interface regions (see also Figures 3 and 4 ). As discussed by Jackman et al. (2019), much of this failure to identify very low values of $P d$ may be due to the fact that for low $P d$, the magnetopause is very expanded, so the Cassini orbit rarely enters the magnetosheath under such conditions.

\subsection{Comparison With $P d$ Inferred From Magnetopause and Bow Shock Crossings}

Recently, Jackman et al. (2019) have presented a comprehensive list of times when Cassini crossed Saturn's bow shock or magnetopause, based primarily on the magnetic field signature but with all crossings within the CAPS data availability time range confirmed with CAPS plasma data. Using models of the magnetopause (e.g., Kanani et al., 2010) and bow shock (e.g., Went et al., 2011), each of these crossing locations can be used to infer the upstream solar wind dynamic pressure. Jackman et al. (2019) have done this calculation for all of their observed boundary crossings and have compared the resulting values of $P d$ with the occurrence distribution derived from the mSWiM model (see, for example, Figure $3 \mathrm{~b}$ above). They found that when normalized to spacecraft dwell time in various locations, the occurrence distribution of $P d$ derived from magnetopause crossings is very similar to that derived from bow shock crossings, and both tend to be about a factor of 2 higher than the corresponding mSWiM distribution.

Figure 6 shows the occurrence distributions of $P d$ inferred from our magnetosheath parameters (equation (10)) and from the observed locations of the magnetopause and bow shock crossings cataloged by Jackman et al. (2019) (using the models of Kanani et al. (2010) and Went et al. (2011)). Because we are 


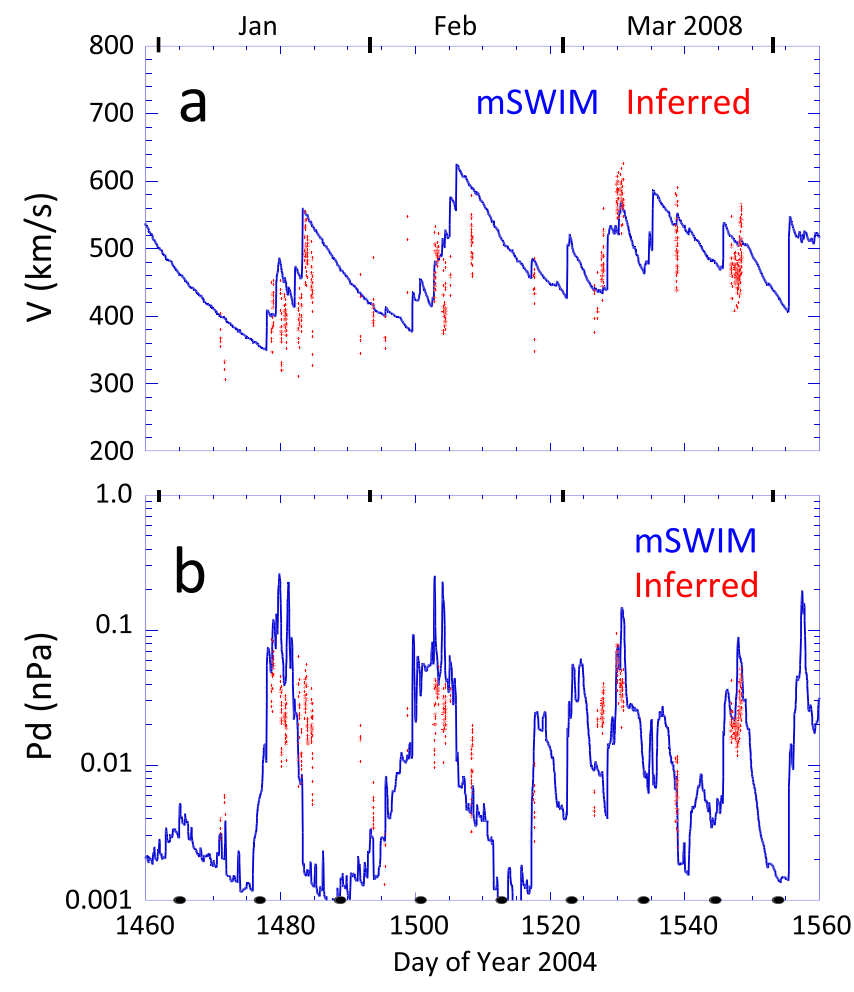

Figure 5. Same as in Figure 4 but for just the first three months of 2008, during which time the Earth and Saturn were near opposition, so the mSWiM predictions are expected to be particularly good. For reference, day 1 of 2008 is day 1,462 of 2004.

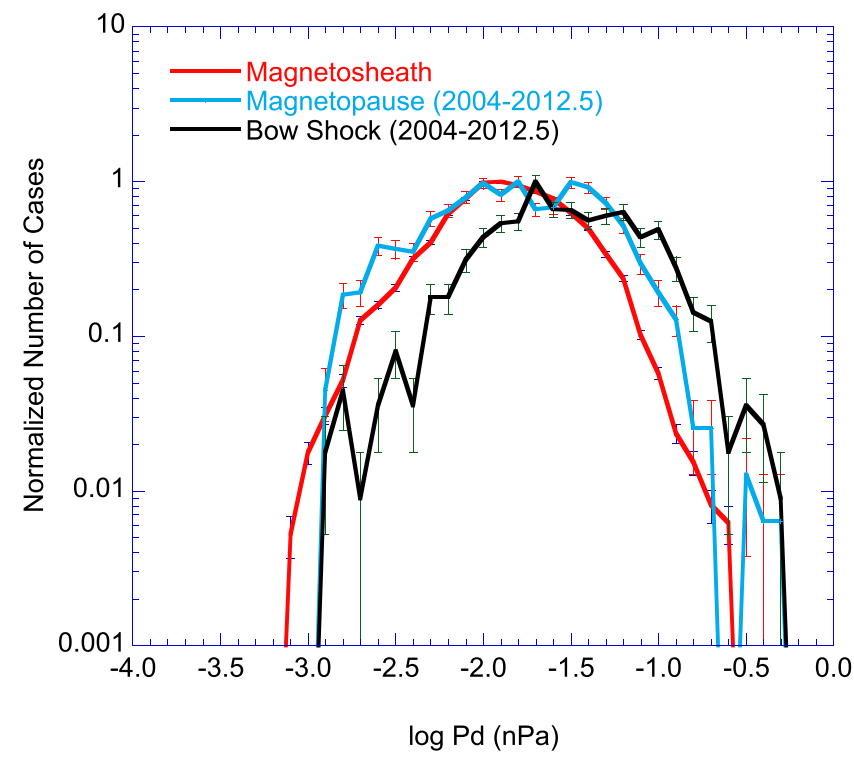

Figure 6. Normalized occurrence distribution of solar wind dynamic pressure estimated from magnetosheath parameters (red); from observed locations of magnetopause crossings, combined with the magnetopause model of Kanani et al. (2010) (light blue); and from observed locations of bow shock crossings, combined with the bow shock model of Went et al. (2011) (black). The magnetopause and bow shock crossings were identified by Jackman et al. (2019). comparing with the CAPS-derived values, only the boundary crossings up to the end of CAPS data in mid-2012 are included. For this figure, no normalization for spacecraft dwell time has been done for either set of boundary crossings.

In Figure 6 it is first noteworthy that unlike the case when normalization for spacecraft dwell time is done (Jackman et al., 2019), the occurrence distributions derived from the magnetopause and bow shock crossings are somewhat different. The distribution based on bow shock crossings is shifted toward higher $P d$ than that from the magnetopause crossings. At the low dynamic pressures, this is consistent with the orbital bias discussion of Jackman et al. (2019): a low value of $P d$ would move the boundaries to larger radial distances, especially for the bow shock, taking them beyond typical Cassini apogee (c.f., Figure 6 of Jackman et al. (2019)). The normalization for spacecraft dwell time removes this low-Pd offset (c.f., Figure 7 of Jackman et al. (2019)). At high $P d$ the slight offset $(\sim 1.5 \mathrm{X})$ is less easily explained and persists even when the occurrences are normalized (c.f., Figure 7 of Jackman et al. (2019)).

The occurrence distribution of $P d$ values derived from magnetosheath parameters (blue curve, Figure 6) must be similarly affected by orbital coverage, and one would expect the distribution to suffer some reduction at low $P d$ compared to the bow shock, but less than for the magnetopause, consistent with what is found in Figure 6. On the other hand, at high $P d$ the magnetosheath distribution shows substantially lower occurrence than either the bow shock or magnetopause distributions. There seems to be no obvious reason why this should be the case.

When magnetosheath measurements are made near a magnetopause or bow shock crossing, we would expect that the values of $P d$ inferred from the plasma measurements and from the boundary locations should be similar. Figure 7 explores this relationship for the set of magnetosheath points that were obtained within $100 \mathrm{~min}$ of a boundary crossing in the Jackman et al. (2019) data set (magnetopause in Figure 7a and bow shock in Figure 7b). The expected correlation is clear in both panels, but the values of $P d$ obtained from the magnetosheath data are commonly smaller than those obtained from the boundary crossings. The median ratio of $P d$ from the magnetosheath to $P d$ from the magnetopause location is 0.62 , with $90 \%$ of the values between 0.14 and 2.26 . For the bow shock location the median ratio is 0.58 , with $90 \%$ of the values between 0.23 and 1.29 . Thus, on the average it appears that the dynamic pressure we derive via equation (10) is about a factor of 2 lower than one would infer from nearby boundary crossings.

\subsection{Comparison With Pd Bounds From Magnetopause and Bow Shock Models}

While measurements in the near vicinity of known boundary crossings provide a very useful comparison for the results of the procedure developed above, it is also possible to use the boundary models themselves (e.g., Kanani et al., 2010; Went et al., 2011) to set local limits on the value of $P d$ for each magnetosheath data point. This can be done quite simply by noting that at any given point, in order for the spacecraft to be in the magnetosheath there, the upstream dynamic pressure must be larger than would be needed to bring the magnetopause to that point but smaller than what would be needed to bring the bow shock there. Thus, we would 

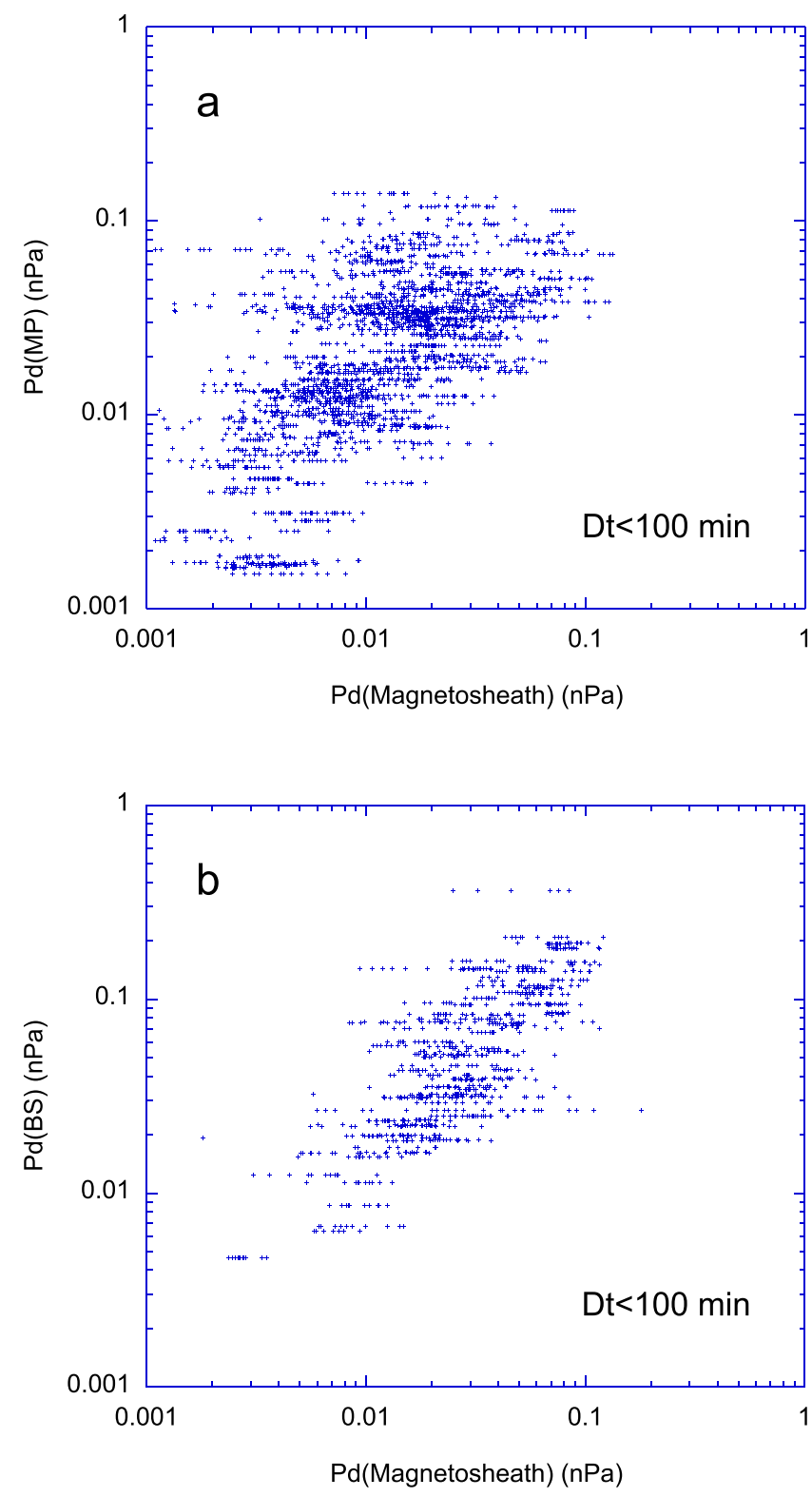

Figure 7. Solar wind dynamic pressure estimated from magnetosheath parameters (horizontal axis) compared with (a) $P d$ inferred from the Kanani et al. (2010) magnetopause model applied to observed magnetopause crossings and (b) inferred from the Went et al. (2011) bow shock model applied to observed bow show crossings. The magnetopause and bow shock crossings are from the catalog of Jackman et al. (2019), and only magnetosheath points within 100 min of an observed boundary crossing are included in the plot. expect the dynamic pressure inferred from magnetosheath parameters to lie between these two limits.

Figure 8 shows several examples of detailed comparisons between the values of $P d$ inferred from magnetosheath parameters (red) and the values that would be required to put the magnetopause at that location according to the Kanani et al. (2010) model (dark green) and the bow shock according to the Went et al. (2011) model (light purple). In addition, the bright green and blue open circles show the values of $P d$ inferred from observed magnetopause and bow shock crossings (Jackman et al., 2019) according to the same models. Our expectation is that the red symbols should generally lie between the dark green and purple curves. For the intervals shown in Figures $8 \mathrm{a}$ and $8 \mathrm{~b}$, this is the case, with a few exceptions. Moreover, the magnetosheath points nicely fill the region between observed magnetopause and bow shock crossings. By contrast, Figure 8c shows an interval where the $P d$ inferred from magnetosheath measurements lies well below the expected zone between local magnetopause and bow shock. Figure 8d shows another interval where the inferred $P d$ generally lies within the predicted magnetosheath range. Figures $8 \mathrm{e}$ and $8 \mathrm{f}$ show two subintervals from within the broader time range covered by Figure $8 \mathrm{~d}$. In Figure $8 \mathrm{e}$ the inferred $P d$ progresses from near the magnetopause values at $\sim$ day $1,278.35$, at which time there were numerous observed magnetopause crossings, to near bow shock values late in day 1,279 , at which the spacecraft was observed to cross the bow shock 3 times. The inferred $P d$ thus is in excellent accord with the sequence of boundary crossings. A similar situation is illustrated in Figure 8f, where the reverse progression occurs: shortly after the inbound bow shock crossing early on day 1,290, the inferred $P d$ was near the bow shock value; thereafter, it declined to values near the magnetopause prediction, at which point two magnetopause crossings were actually observed. Thus, except for Figure 8c, the intervals in Figure 8 show very satisfying correspondence between our calculated values of $P d$ and the model magnetosheath range.

The mismatch shown in Figure 8c is unfortunately not unique in the data set. Figures $9 \mathrm{a}$ and $9 \mathrm{c}$ show the ratios of $P d$ derived from magnetosheath parameters to the values predicted for the magnetopause and bow shock, respectively. As argued above, the ratio should be greater than 1 for the magnetopause and less than one for the bow shock. The latter condition seems to be well satisfied by almost all the measurements (Figure 9c), but $P d$ inferred from the magnetosheath measurements is frequently below the value predicted for the magnetopause (Figure 9a). This suggests that, if the Kanani et al. (2010) model correctly relates $P d$ to the magnetopause location, the magnetosheath estimation sometimes $(\sim 35 \%$ of the time) is too low, consistent with the conclusion drawn above in the discussion of Figure 7. However, as clearly seen in the Figure 8 panels other than Figure 8c, this underestimate is only episodic, and there are many intervals where the values appear to be in good accord with the model magnetopause and bow shock bounds.

Figures $9 \mathrm{a}$ and $9 \mathrm{c}$ indicate that there is a systematic temporal variation in times when $P d$ appears to be underestimated. Systematically lower values seem to occur primarily between about days 2,200 and 2,500. It turns out that this time interval was also when the Cassini apogee was at its greatest local time (see for example Figure 2c of Thomsen et al., 2018). Figures 9b and 9d examine a possible relationship with local time. While there are numerous measurements across the full range of LT where the magnetosheath-tomagnetopause ratio falls below 1, there does seem to be a systematic sag to lower values beyond $\sim 18$ LT. There is a similar region of lower ratios at local times before 5 (but with some notable exceptions). It may 

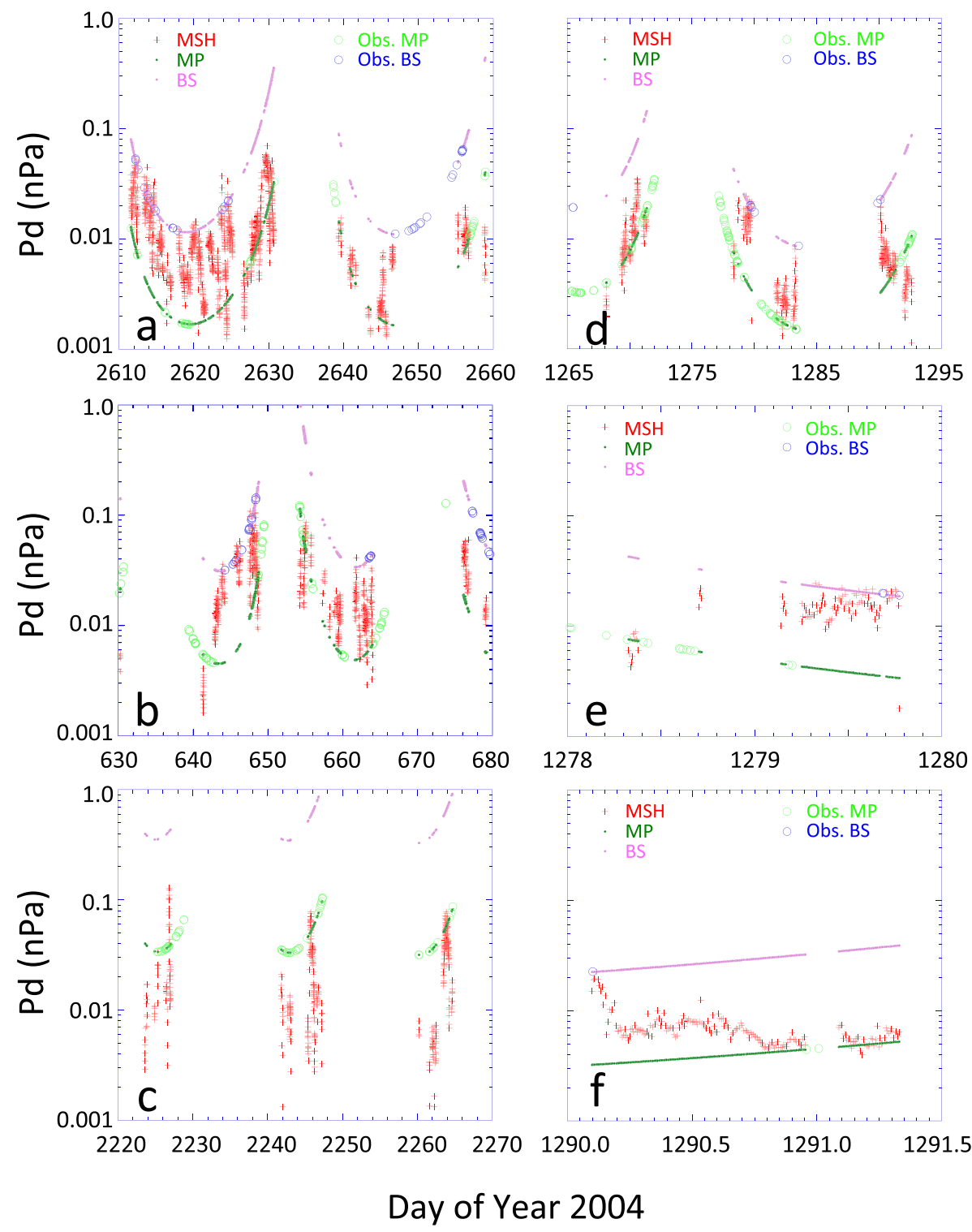

Figure 8. Solar wind dynamic pressure estimated from magnetosheath parameters (red symbols) compared with $P d$ inferred from the Kanani et al. (2010) magnetopause model applied to the local spacecraft position (dark green symbols) and the Went et al. (2011) bow shock model applied to the local spacecraft position (purple symbols). Also shown are the $P d$ inferred from observed magnetopause and bow shock crossings (Jackman et al., 2019), determined using the same two models. Since the red symbols are determined from actual magnetosheath measurements, they should lie between the curves for the magnetopause and bow shock at the spacecraft position. For reference, the beginning dates of the six panels are (a) 2011 day 53, (b) 2005 day 264, (c) 2010 day 28, (d) 2007 day 169, (e) 2007 day 182, and (f) 2007 day 194.

thus be that the analysis outlined in section 3 becomes less appropriate for local times beyond the terminator, and it is probably reasonable to exercise caution when using those values. As for other times where the ratio falls below 1 , some may be attributable to density fluctuations in the magnetosheath, but other systematic departures are currently unexplained.

\subsection{Comparison With SKR}

In the introduction to this study, established evidence relating SKR activity to the upstream dynamic pressure was noted. Using the RPWS database of 3-min averaged SKR flux densities between 100 and $300 \mathrm{kHz}$ observed by Cassini throughout the time interval covered by the CAPS data, we have compared the SKR fluxes with the dynamic pressure inferred from the magnetosheath measurements. We have surveyed all 

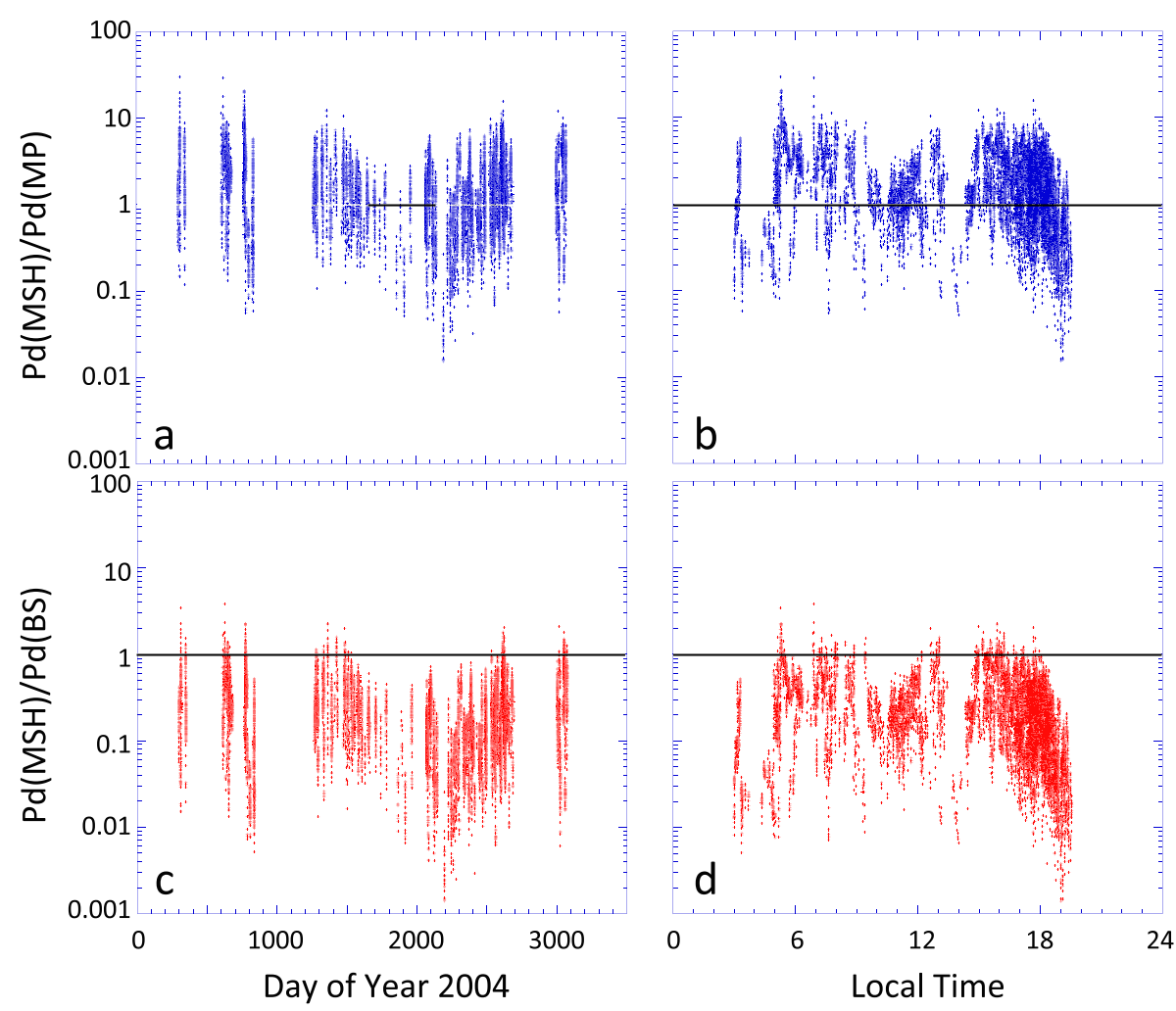

Figure 9. The ratio of the solar wind dynamic pressure estimated from magnetosheath parameters to that inferred from (a and b) the Kanani et al. (2010) magnetopause model applied to the local spacecraft position and (c and d) the Went et al. (2011) bow shock model applied to the local spacecraft position. Figures 9a and 9c show the ratios plotted as a function of day of year 2004, and Figures $9 \mathrm{~b}$ and $9 \mathrm{~d}$ show them plotted as a function of local time.

several-day intervals during which there was good modulation of the inferred dynamic pressure. In particular, we sought times when the dynamic pressure rose or fell significantly. These were then compared with the corresponding SKR fluxes.

Figure 10 shows the data for an eight-day interval where good correspondence was observed. These observations were obtained within a local time range of 2.85-3.66 LT and a latitude range of $0.15-0.07^{\circ}$, where we would expect good SKR source visibility (Kimura et al., 2013; Lamy et al., 2008). Figure 10a shows the upstream solar wind speed inferred using equation (8). Figure 10b shows the inferred dynamic pressure (equation (10), red symbols) superimposed on the electron density measured by CAPS/ELS (light blue symbols). Also shown as the large black open circles are values of $P d$ inferred from actual magnetopause crossings (again using the model of Kanani et al. (2010)). Finally, Figure 10c shows the SKR fluxes. Figure 10b emphasizes the fact that the dominant contribution to variations in the upstream dynamic pressure is from the density. The fact that electron density measurements exist in intervals when there are not dynamic pressure values is due to the fact that the electrons are subsonic in the magnetosheath, and therefore, the orientation of the spacecraft is largely irrelevant to the measurement, allowing electron density determinations when ion measurements are not possible due to poor viewing. Prior to 1,500 UT on day 832 and on and off after $\sim 2,000$ UT on day 836, Cassini was in the high-latitude plasma sheet or lobe, where the density is quite low.

Figure 10 shows that during this eight-day interval there were order-of-magnitude variations in both dynamic pressure and SKR fluxes. Indeed, this time period encompasses one of the long-lasting low-frequency-extension (LFE) SKR events identified by Reed et al. (2018) as being related to solar wind $P d$ enhancements. Figure 7 of that paper shows that a solar wind $P d$ enhancement was predicted by mSWiM to occur early on 2006 day 100, approximately two days prior to this LFE event, but Figure 10 above shows that that enhancement actually arrived two days later than the mSWiM prediction, in very good agreement with the onset of the LFE event, thereby strengthening the association deduced by Reed et al. (2018). 


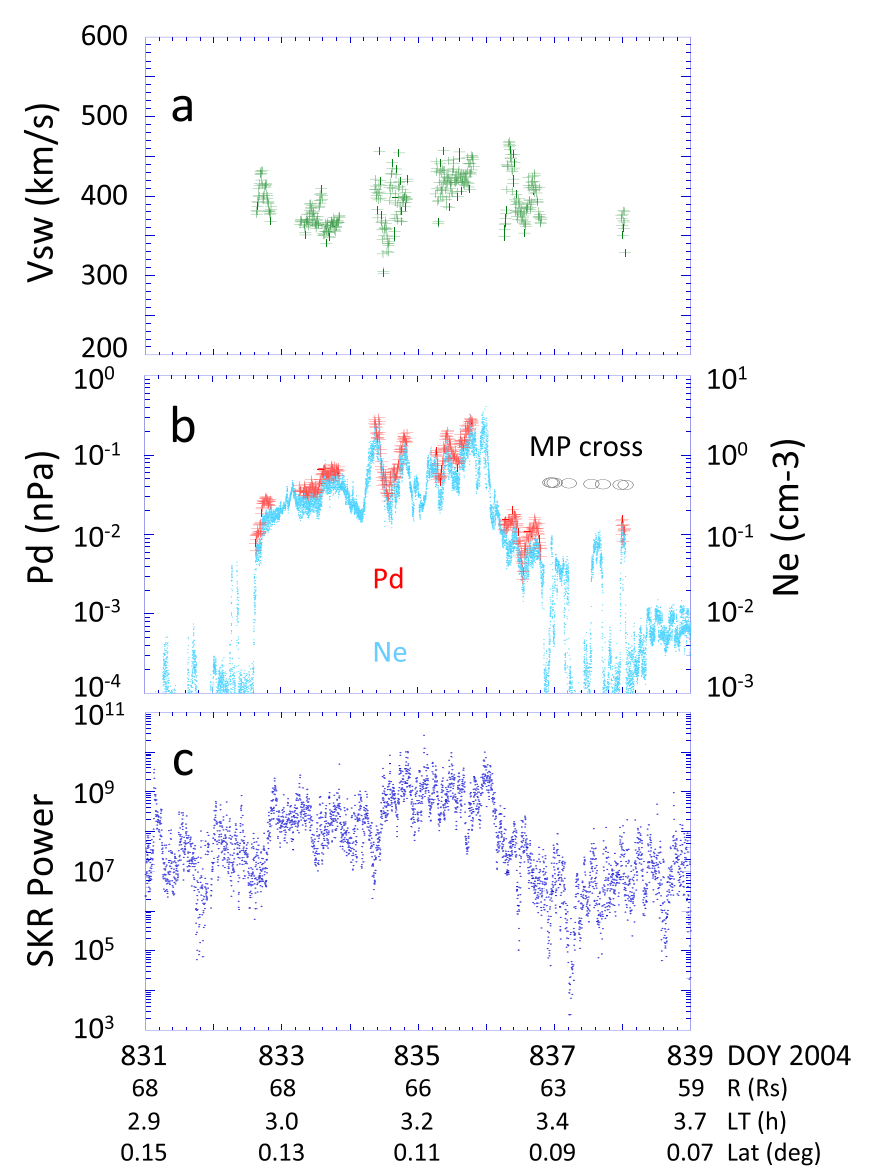

Figure 10. (a) Solar wind flow speed and (b) dynamic pressure (red) estimated from magnetosheath parameters, (c) compared to measured SKR flux densities (in $\mathrm{Wm}^{-2} / \mathrm{Hz}$ ) averaged between 100 and $300 \mathrm{kHz}$ derived from Cassini/RPWS measurements. Also shown in (b) are the dynamic pressures estimated from observed magnetopause crossings (open ovals) and the electron density at Cassini measured by the CAPS/ELS instrument (light blue). The date range spanned by this eight-day interval is from 2006 day 100 (10 April) through day 107 (17 April), when Cassini was inbound near $3 \mathrm{LT}$, between $\sim 59$ and $68 R_{S}$, very near the equatorial plane.
Comparison of the three panels in Figure 10 suggests a rather close correspondence between the SKR fluxes and the dynamic pressure, but less so for the inferred solar wind speed. Figure 11a explores the correspondence in more detail. In that figure, the SKR fluxes have been smoothed with a running 10-point average and translated in time to better match the temporal variations of $P d$. The time translation is a 4-hr delay for the SKR relative to the magnetosheath measurements. The black crosses near the top of the figure indicate the times when the SKR phase is zero, as calculated with the fit derived by Kurth et al. (2007). These points would be about an hour earlier if we used the SKR $S$ phase system of Lamy et al. (2011), as appropriate for 2006, when the southern system was dominant. Zerophase times correspond to predicted peaks in the SKR intensity. Like the SKR fluxes themselves, these crosses have been translated to the left by $4 \mathrm{hr}$. For this particular interval, there is substantial variability of the SKR on the time scale of hours or less, but the peaks do not seem to be well associated with the predicted zero-phase times.

In Figure 11 the relationship between the SKR fluxes and the solar wind dynamic pressure is clear, but not entirely one-to-one. Significant increases in $P d$ are accompanied by similar increases in SKR (e.g., day 832.6, 834.3), and the SKR is generally more intense when the pressure is high and orders of magnitude less intense when the pressure is low, as, for example, on day 836 (when the dynamic spectrum, not shown, actually shows a brief complete extinction of the radiation). However, there is short-term (less than few hours) structure in SKR that does not track short-term variations in $P d$. Note that the SKR is plotted with two decades for each decade of $P d$ variation, suggesting the possibility that the magnitude of SKR fluxes may be related to the square of the dynamic pressure. The magnetopause crossing near the end of day 836 occurs when the dynamic pressure is notably low, that is, during a time of magnetospheric expansion and low SKR.

The good correspondence between SKR fluxes and $P d$ seen in Figure 11 is not common in this data set. Figure 12, in the same format as Figure 11, shows a sampling of four other intervals in which there were inferred $P d$ values available over several days, and during which there was appreciable temporal modulation of $P d$. Some intervals show reasonably good correspondence, but others show little if any.

Figure 12a, plotted with a 4-hr delay as for the event in Figure 11, shows some correspondence especially on day 310. Bow shock crossings (black crosses) generally appear to bracket SKR enhancements (outbound during SKR increases; inbound during SKR decreases). Similarly, outbound magnetopause crossings (black open circles), consistent with increasing $P d$, tend to occur during SKR enhancements, and inbound crossings more likely during decreasing SKR fluxes.

Figure $12 \mathrm{~b}$ shows a longer interval, with no temporal offset imposed. For this 12-day time period, there appears to be quite good correspondence between the large-scale variations in $P d$ and SKR fluxes. The generally lower fluxes in the first three days of the interval happen during very low values of $P d$, with a jump up in fluxes on day 773 clearly associated with a tenfold increase in the estimated $P d$. The very large spike in SKR fluxes on day 781 at the end of the interval is preceded by a large increase in electron density (and presumably $P d$, given the good tracking of the two) just prior to the outbound bow shock crossing. Close examination of the timing of this increase suggests that the rise in SKR followed the density enhancement by less than $2 \mathrm{hr}$. Examination of the low-Pd interval on days 771 and 772 shows a reasonably good tracking between the dynamic pressure variations and the SKR variations, with a time lag of $\sim 5 \mathrm{hr}$.

Figure 12c shows some correspondence in the second half of the 10-day interval, except that the steep enhancement in SKR on day 2,558 occurred in coincidence with an inbound magnetopause crossing, 


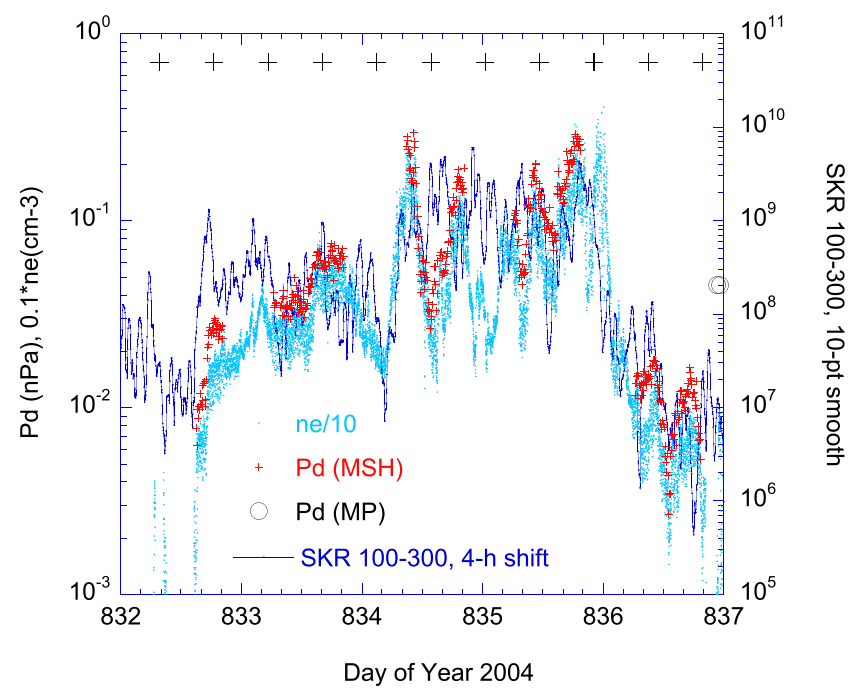

Figure 11. Solar wind dynamic pressure (red), estimated from magnetosheath parameters, and local magnetosheath electron density (light blue), compared to measured SKR fluxes between 100 and $300 \mathrm{kHz}$ (10-pt smoothed, every tenth point, dark blue). Also shown are the dynamic pressures estimated from observed magnetopause crossings (open circles). Measurements are from 2006 day 101 (11 April) through day 105 (15 April), while the spacecraft was near 3 LT. SKR measurements are plotted with a 4 hour shift to the left. Black crosses at the top indicate the (4-hr shifted) times with SKR phase of zero, according to the formulation of Kurth et al. (2007). These are the predicted maxima in the SKR intensity. attributable to the sharply declining $P d$. During the first half of the interval in Figure 12c, there is no discernible correspondence. Indeed, the highest SKR fluxes occurred during times of very low $P d$, and like the one at the end of the plot, the upward spike in SKR in the first half of day 2,551 occurred near an inbound magnetopause crossing, due to decreasing solar wind dynamic pressure.

Figure 12d shows essentially no correspondence between SKR and $P d$. The rise in emissions coincides more with a declining dynamic pressure, and the spike on day 3,058 occurs during a sharp minimum in the inferred $P d$. During this interval, and indeed for all four of the intervals shown in Figure 12, there is little evidence of a $10.6-\mathrm{hr}$ periodicity in the SKR flux enhancements.

The intervals in Figures 11 and 12 show that the relationship between SKR enhancements and solar wind dynamic pressure is far from simple. While there are times when the average SKR flux tracks $P d$ with some fidelity for days (e.g., Figures 11 and 12b), there are other times when there is essentially no correlation. One factor relevant to whether or not a relationship is seen is the local time of the observation: in our survey of intervals to highlight for this report, the few predawn events showed the best relationship (Figures 11, 12a, and 12b), whereas the events beyond 11 LT almost all showed questionable if any correspondence. As shown by previous authors (as summarized, e.g., by Lamy (2017)), SKR is strongly beamed into a thin hollow cone, and the location of the source region Cassini can see at any given time depends on the spacecraft location. When Cassini is close to the equator, the spacecraft preferentially detects radio sources along field lines whose LT differs by less than a few hours from the subspacecraft meridian. Our finding indicates that the SKR source is most responsive to $P d$ variations in a region primarily visible from the morning region of the magnetosphere, which is the location of the dominant source region. The secondary (afternoon/dusk) sources are thus not as clearly driven by $P d$. In fact, as shown above there are several examples where an increase in SKR appeared to follow a significant decrease in $P d$.

\section{Summary and Discussion}

The primary objective of this study has been to introduce a method for estimating upstream solar wind properties (dynamic pressure and flow speed) from observations of magnetosheath parameters at Saturn. The analytical method takes advantage of the high plasma beta in Saturn's magnetosheath, as well as the high Mach number of the bow shock, to approximate the magnetosheath flow with a hydrodynamic approach that neglects the dynamic influence of the magnetic field. While 95\% of magnetosheath measurements have a plasma beta greater than 1 (Thomsen et al., 2018), the magnetic field may in fact contribute significantly to the dynamics in a fraction of cases. In particular, the neglect of small magnetic forces applied systematically over the flow path might lead to inaccuracies in the results of our analytical approach. This possibility could be examined with global MHD simulations of the Saturn/solar wind interaction, but it is beyond the scope of the present study.

Other uncertainties is the calculation of $P d$ and $V_{\mathrm{sw}}$ are introduced by assumptions that are needed to estimate the point at which a given magnetosheath parcel crossed the bow shock (equation (15)), as well as regarding the Mach number dependence of the relation between upstream and downstream flow angles relative to the normal (equation (12)). As argued above, we believe these assumptions probably make no more than a factor of 2 uncertainty in the resulting value of $P d$. Another significant issue for the method presented here is whether or not to include the energetic particles in the plasma pressure needed for equation (10). Such particles are introduced into the magnetosheath by way of leakage from the magnetosphere, so they are not really included in the conservation equations (equations (1) and (2)) linking upstream properties to downstream properties. Nonetheless, we have done an empirical comparison of the 


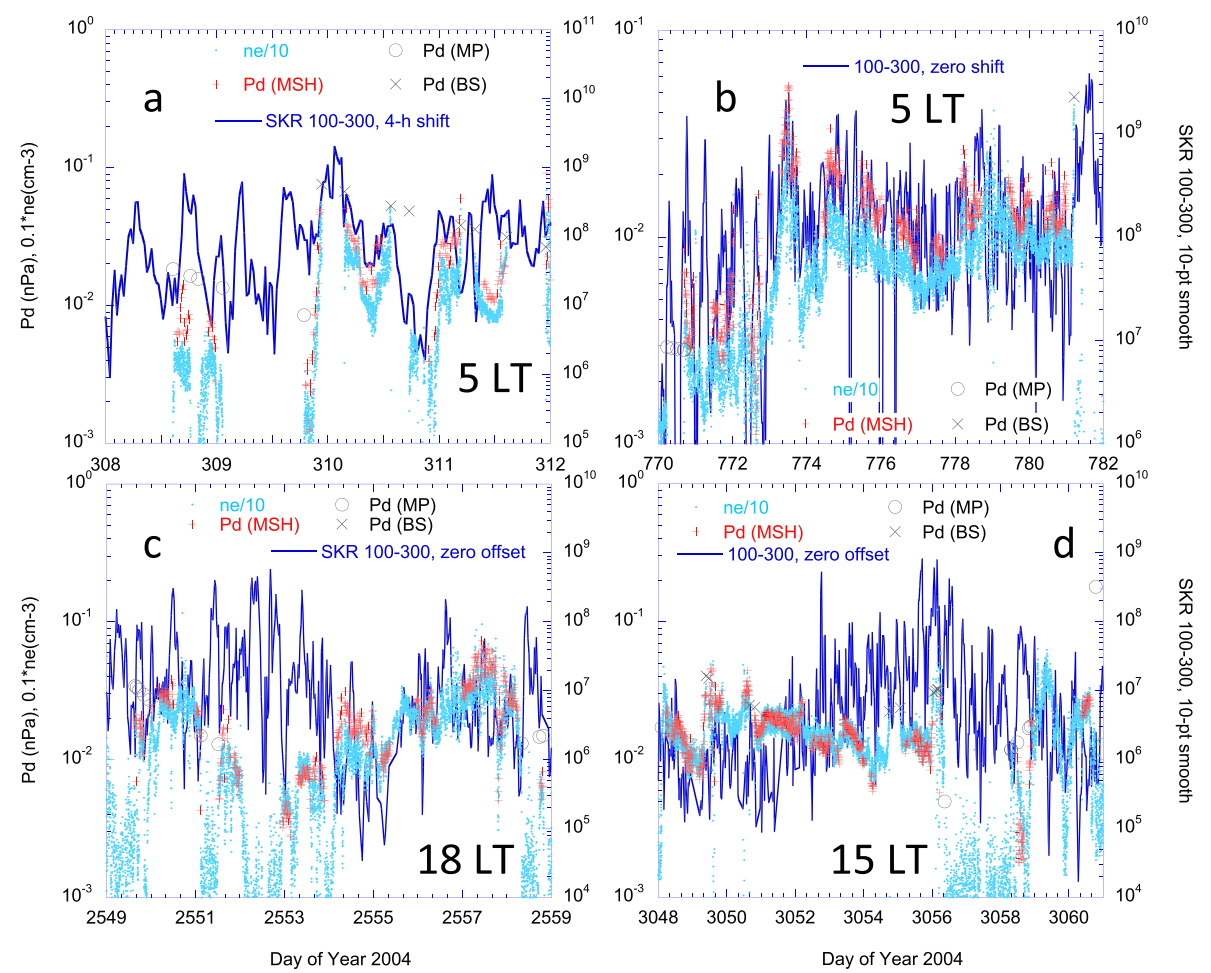

Figure 12. Same as in Figure 11 for four other time periods with fairly lengthy intervals of inferred dynamic pressure: (a) 2004 days 308-312, (b) 2006 days 39-51, (c) 2010 day 357-2011 day 2, and (d) 2012 days 126-139. The black cross symbols indicate estimated dynamic pressures from observed bow shock crossings. In (a) SKR measurements are plotted with a 4-hr shift to the left; other panels have no temporal offset. Figures 12a and 12b were obtained near $5 \mathrm{LT}$, Figure $12 \mathrm{c}$ near 18 LT, and Figure 12d near 15 LT. For clarity, in all panels only every third electron density point is plotted, and only every tenth SKR point.

distribution of $P d$ and $V_{\text {sw }}$ predicted at Saturn's orbit by the mSWiM MHD calculation and estimated both with and without the energetic particle contribution to the pressure. The effect of on the calculation of $P d$ is quite modest, but including the energetic particles creates a high- $V_{\mathrm{sw}}$ tail that is clearly missing in the mSWiM distribution (Figure 3a), so on the basis of this finding, we have not included the energetic particle pressure in our estimates of $P d$ and $V_{\text {sw. }}$.

One other significant source of quantitative uncertainty in the method described here is the adopted value of the polytropic index. We have used $\gamma=5 / 3$, but observations at the Earth's bow shock have found generally lower values, with the most probable value $\sim 1$ (isothermal), and the distribution extending down to $\sim 0$ (isobaric; Park et al., 2019). The formalism described above does not allow values $\gamma \leq 1$, but we have explored the consequences of using values smaller than $5 / 3$. We find that for $\gamma=4 / 3$, the estimated solar wind speed $V_{\mathrm{sw}}$ is on average $\sim 16 \%$ higher than for $5 / 3$, with the result that the peak in the occurrence distribution matches that of mSWiM very well. For $\gamma=1.1$, the estimated $V_{\mathrm{sw}}$ is $70 \%$ higher, and the distribution becomes much broader, with a long high- $\mathrm{V}$ tail. The effect of $\gamma$ on the derived values of $P d$ is more complicated because $\gamma$ enters two terms in equation (10): the square root term and the ratio of cosines. The square root term is exactly the same as the $\gamma$-dependence of $V_{\mathrm{sw}}$, producing average increases in $P d$ in the neighborhood of $16 \%$ for $\gamma=4 / 3$. However, the ratio of cosines has a $\gamma$ dependence (see equation (12)) that counteracts the effect of the square root term. In fact, for $\gamma=4 / 3$, the cosine ratio decreases by a factor of up to 1.7 , so the two $\gamma$-dependent terms in equation (10) largely cancel (and at even smaller $\gamma$, the decrease from the cosine ratio dominates). Determination of the polytropic index in Saturn's magnetosheath is beyond the scope of the current study, but it seems possible that use of a slightly smaller value than $5 / 3$ in the present formalism might improve the statistical match with the mSWiM solar wind speed, while not greatly affecting the derived $P d$. 
In sum, the uncertainties associated with the issues listed above can probably account for the rather small discrepancies between our estimated values of $P d$ and the various comparison values we described above and summarize below.

We have sought to validate our derived values of $P d$ and $V_{\text {sw }}$ by comparing them with mSWiM predictions, both on a statistical basis and on a time-profile basis. In general, we find good correspondence between the two sets of values. The magnetosheath-derived parameters have more point-to-point variability, presumably due in part to fluctuations introduced in the foreshock, shock, or magnetosheath itself, and also due to the MHD smoothing of small-scale structures over the long propagation distance from 1 A.U. to Saturn. Longand short-term temporal variability is reasonably well reproduced, with $P d$ and $V_{\mathrm{sw}}$ increases typically identified within a few days of their predicted arrival by mSWiM.

Our inferred $V_{\mathrm{sw}}$ is statistically about $40 \mathrm{~km} / \mathrm{s}$ lower than the $\mathrm{mSWiM}$ predictions. For $P d$ the principal discrepancy in the comparison is that we find far fewer low values of $P d\left(10^{-3}-10^{-2} \mathrm{nPa}\right)$ than predicted by mSWiM. As noted by Jackman et al. (2019), very low values of $P d$ would be less likely to be observed in magnetosheath measurements because under those conditions the magnetopause expands out beyond Cassini's orbital range. On the other hand, the observations compiled by Jackman and Arridge (Figure 3 above) should exhibit no such bias, and they also show a lack of low-Pd measurements compared to the mSWiM distribution. Of course, the observations summarized by Jackman and Arridge were obtained primarily near solar maximum, when the interplanetary medium rarely displays the compression/rarefaction signature of interacting streams that is more prevalent during the declining phase of the solar cycle and appears to have characterized the interval examined in Figure 5. Thus, the question of the prevalence of low- $P d$ intervals in those rarefaction regions at Saturn's distance remains to be addressed, and a comparison between the mSWiM values and the predictions of other MHD models (e.g., Pogorelov et al., 2014; Tao et al., 2005) would seem warranted.

Comparison of the magnetosheath-inferred values of $P d$ with those estimated from the locations of magnetopause and bow shock crossings (Jackman et al., 2019) shows that both statistically and on a case-by-case comparison with measurements made within $100 \mathrm{~min}$ of a boundary crossing, the magnetosheath estimates tend to be on average about a factor of 2 lower than the boundary location determinations.

Similarly, the values of $P d$ estimated from magnetosheath parameters are generally well bounded on the high end by the values that would be required to bring the bow shock to the observation point (according to the Went et al., 2011, model), but there are many occasions when the estimated $P d$ is lower than what would be required to bring the magnetopause out to the observation point (according to the Kanani et al., 2010, model; see Figure 9). This deficiency in the estimated dynamic pressure is particularly pronounced at local times beyond the terminator and may point to an inadequacy in the analytical methodology at large angles from noon.

Finally, the estimated values of $P d$ have been compared with temporal sequences of 3-min averages of SKR flux density averaged over 100-300 kHz observed by Cassini. We sought in particular all several-day intervals during which there was good modulation of the inferred dynamic pressure. By focusing on relatively large pressure variations, the study examined intervals that were more likely to feature the long-lasting solar wind -driven events discussed by Lamy et al. (2018) and Reed et al. (2018). Indeed, the interval featured in Figures 10 and 11 was one of the long-lived LFE events identified by Reed et al. (2018).

We found a number of episodes where there appeared to be good tracking between the inferred $P d$ and the SKR intensity on the time scale of more than a few hours. During these intervals, sharp increases (decreases) in $P d$ correspond to significant increases (decreases) in SKR intensity, and generally high (low) values of $P d$ correspond to generally high (low) values of SKR. At times the tracking seems to show large-scale SKR variability that goes roughly as the square of the dynamic pressure variations, with temporal delays $\sim 4 \mathrm{hr}$ between $P d$ and SKR. For observation points in the dawn sector, where the best correspondence between SKR and $P d$ is found, this corresponds to a time delay of $\sim 11-12 \mathrm{hr}$ from the time the solar wind structure arrived at the nose of the magnetopause, assuming an average magnetosheath flow speed of $\sim 150 \mathrm{~km} / \mathrm{s}$ (c.f., Figure $5 \mathrm{~d}$ of Thomsen et al., 2018) and a typical magnetopause standoff distance of $26 R_{S}$ (Figure $5 \mathrm{~h}$ of Jackman et al., 2019). This is comparable to the time delays reported by Taubenschuss et al. (2006) and Badman et al. (2008). The relatively short time delays following the transport of the structures to Saturn's nightside 
magnetosphere suggest that no large growth phase is required to build up the conditions needed for SKR enhancement. Rather, some of the events we have identified indicate a rather directly driven influence.

Such is not the case, however, for the intervals observed while the spacecraft was at local times beyond $\sim 10$ LT. In those regions, tracking between $P d$ and SKR was less clear. Indeed, there are several examples where an increase in SKR appeared to follow a significant decrease in Pd. One possible explanation is that the energized electrons that produce SKR (e.g., Kurth et al., 2009; Menietti et al., 2011; Schippers et al., 2011) arise from dynamical events (especially magnetic reconnection) associated with the stretching and subsequent dipolarization of magnetospheric magnetic field lines (e.g., Jackman et al., 2009). On the nightside, enhanced solar wind pressure strengthens the equatorial current sheet, increasing the field line stretching, and triggering tail reconnection (e.g., Jackman et al., 2010). On the dayside, Delamere et al. (2015) have suggested that smaller-scale magnetic reconnection is also a common occurrence. There, however, enhanced solar wind pressure pushes the magnetosphere into a more dipolar shape (Arridge et al., 2008), reducing the stretching and making the system more resistant to reconnection. Relaxation of the pressure may thus increase the stretching and lead to enhanced reconnection and emission, as noted above in Figures 12c and $12 \mathrm{~d}$.

\section{Conclusions}

An analytical method has been presented (equations (8) and (10)), by which the large database of Cassini measurements from within Saturn's magnetosheath can be exploited to estimate the upstream solar wind conditions, namely, the dynamic pressure and the solar wind speed. The results have been compared statistically and by detailed time sequence with other measures of those parameters. These include the dynamic pressure and solar wind speed predicted at Saturn's orbit by the mSWiM MHD model, the dynamic pressure inferred by fitting magnetopause and bow shock models to observed crossings of those boundaries, and the bounds imposed by those same models on the dynamic pressures needed to place the magnetopause or bow shock at a given observation point. The results are generally in good agreement with these other estimates, although the magnetosheath-estimated values on average seem to underestimate the upstream flow speed by $\sim 40 \mathrm{~km} / \mathrm{s}(\sim 10 \%)$. The dynamic pressures also seem to be underestimated slightly, by up to a factor of 2 , especially at local times tailward of the terminator. These relatively small discrepancies are within the uncertainties inherent in the analytical method. The inferred values of $P d$ do not extend to the low values expected from mSWiM, probably at least in part because the magnetosheath would not be observable under such major magnetospheric expansions.

This work emerged from collaborative discussions that took place during a visit of MFT to the University of Southampton as a Diamond Jubilee Fellow. The support provided by the Diamond Jubilee Fellowship is gratefully acknowledged. Work at PSI was supported by the NASA Cassini program through JPL contract 1243218 with Southwest Research Institute. The Cassini project is managed by the Jet Propulsion Laboratory for NASA. C.M. J. is supported by STFC Ernest Rutherford Fellowship ST/L004399/1, and L.L. was supported by CNES and national programs of planetology and heliophysics of CNRS/INSU. All Cassini plasma and energetic-particle data used for this study are available from the electronic supplement to Thomsen et al. (2018) or more generally from the Planetary Data System (http://pds.nasa. gov/). The necessary satellite ephemeris data are also available from the same PDS site. The mSWiM predictions of solar wind properties are publicly available on the University of Michigan Web site (http://mswim.engin.umich. edu/). We thank K.C. Hansen and B. Zieger for providing these model results. Cassini/RPWS SKR processed data are available through the Kronos database at http://lesia.obspm.fr/ kronos.
The estimated values of $P d$ have also been compared with temporal sequences of 3-min averages of SKR fluxes observed by Cassini during all several-day intervals in which there was good modulation of the inferred dynamic pressure. At relatively early local times ( $<6 \mathrm{LT})$, there were several examples of noticeably good tracking between SKR and $P d$, with time delays $\sim 4-5 \mathrm{hr}$ between $P d$ structure and corresponding SKR structure. At later local times, the correspondence was rarely persuasive. In light of the visibility constraints on SKR detection, it appears that the SKR source is most responsive to $P d$ variations in a region primarily visible from the morning region of the magnetosphere, which is also the dominant source region. When the correspondence was good (both increases and decreases), the SKR fluxes varied roughly as the square of the dynamic pressure. The generally short time delays between $P d$ and SKR suggest that at times the SKR is rather directly driven by the dynamic pressure.

\section{References}

Arridge, C. S., Achilleos, N., Dougherty, M. K., Khurana, K. K., \& Russell, C. T. (2006). Modeling the size and shape of Saturn's magnetopause with variable dynamic pressure. Journal of Geophysical Research, 111(A11), A11227. https://doi.org/10.1029/2005JA011574

Arridge, C. S., Russell, C. T., Khurana, K. K., Achilleos, N., Cowley, S. W. H., Dougherty, M. K., et al. (2008). Saturn's magnetodisc current sheet. Journal of Geophysical Research, 113(A4), A04214. https://doi.org/10.1029/2007JA012540

Badman, S. V., Bunce, E. J., Clarke, J. T., Cowley, S. W. H., Gérard, J.-C., Grodent, D., \& Milan, S. E. (2005). Open flux estimates in Saturn's magnetosphere during the January 2004 Cassini-HST campaign, and implications for reconnection rates. Journal of Geophysical Research, 110(A11), A11216. https://doi.org/10.1029/2005JA011240

Badman, S. V., \& Cowley, S. W. H. (2007). Significance of Dungey-cycle flows in Jupiter's and Saturn's magnetospheres, and their identification on closed equatorial field lines. Annales de Geophysique, 25(4), 941-951. https://doi.org/10.5194/angeo-25-941-2007

Badman, S. V., Cowley, S. W. H., Lamy, L., Cecconi, B., \& Zarka, P. (2008). Relationship between solar wind corotating interaction regions and the phasing and intensity of Saturn kilometric bursts. Annales de Geophysique, 26, 3641-3651. https://doi.org/10.5194/angeo-26$3641-2008$ 
Badman, S. V., Jackman, C. M., Nichols, J. D., Clarke, J. T., \& Gérard, J. C. (2014). Open flux in Saturn's magnetosphere. Icarus, 231, 137-145. https://doi.org/10.1016/j.icarus.2013.12.004

Badman, S. V., Masters, A., Hasegawa, H., Fujimoto, M., Radioti, A., Grodent, D., et al. (2013). Bursty magnetic reconnection at Saturn's magnetopause. Geophysical Research Letters, 40, 1027-1031. https://doi.org/10.1002/grl.50199

Belenkaya, E. S., Cowley, S. W. H., Badman, S. V., Blokhina, M. S., \& Kalegaev, V. V. (2008). Dependence of the open-closed field line boundary in Saturn's ionosphere on both the IMF and solar wind dynamic pressure: Comparison with the UV auroral oval observed by the HST. Annales de Geophysique, 26(1), 159-166. https://doi.org/10.5194/angeo-26-159-2008

Belenkaya, E. S., Cowley, S. W. H., Nichols, J. D., Blokhina, M. S., \& Kalegaev, V. V. (2011). Magnetospheric mapping of the dayside UV auroral oval at Saturn using simultaneous HST images, Cassini IMF data, and a global magnetic field model. Annales de Geophysique, 29(7), 1233-1246. https://doi.org/10.5194/angeo-29-1233-2011

Blanc, M., Andrews, D. J., Coates, A. J., Hamilton, D. C., Jackman, C. M., Jia, X., et al. (2015). Saturn plasma sources and associated transport processes. Space Science Reviews, 192(1-4), 237-283. https://doi.org/10.1007/s11214-015-0172-9

Bunce, E., Cowley, S. W. H., Talboys, D. L., Dougherty, M. K., Lamy, L., Kurth, W. S., et al. (2010). Extraordinary field-aligned current signatures in Saturn's high-latitude magnetosphere: Analysis of Cassini data during Revolution 89. Journal of Geophysical Research, 115, A10238. https://doi.org/10.1029/2010JA015612

Bunce, E. J., Cowley, S. W. H., Wright, D. M., Coates, A. J., Dougherty, M. K., Krupp, N., et al. (2005). In situ observations of a solar wind compression-induced hot plasma injection in Saturn's tail. Geophysical Research Letters, 32, L20S04. https://doi.org/10.1029/ 2005GL022888

Clarke, J. T., Gérard, J. C., Grodent, D., Wannawichian, S., Gustin, J., Connerney, J., et al. (2005). Morphological differences between Saturn's ultraviolet aurorae and those of Earth and Jupiter. Nature, 433(7027), 717-719. https://doi.org/10.1038/nature03331

Clarke, J. T., Nichols, J., Gérard, J. C., Grodent, D., Hansen, K. C., Kurth, W., et al. (2009). The response of Jupiter's and Saturn's auroral activity to the solar wind. Journal of Geophysical Research, 114(A5), A05210. https://doi.org/10.1029/2008JA013694

Cowley, S. W. H., Badman, S. V., Bunce, E. J., Clarke, J. T., GéRard, J.-C., Grodent, D., et al. (2005). Reconnection in a rotation-dominated magnetosphere and its relation to Saturn's auroral dynamics. Journal of Geophysical Research, 110(A2), A02201. https://doi.org/10.1029/ 2004JA010796

Crary, F. J., Clarke, J. T., Dougherty, M. K., Hanlon, P. G., Hansen, K. C., Steinberg, J. T., et al. (2005). Solar wind dynamic pressure and electric field as the main factors controlling Saturn's aurorae. Nature, 433(7027), 720-722. https://doi.org/10.1038/nature03333

Delamere, P. A., Otto, A., Ma, X., Bagenal, F., \& Wilson, R. J. (2015). Magnetic flux circulation in the rotationally driven giant magnetospheres. Journal of Geophysical Research: Space Physics, 120, 4229-4245. https://doi.org/10.1002/2015JA021036

Desch, M. D. (1982). Evidence for solar wind control of Saturn radio emission. Journal of Geophysical Research, 87(A6), 4549-4,554. https:// doi.org/10.1029/JA087iA06p04549

Desch, M. D. (1983). Radio emission signature of Saturn immersions in Jupiter's magnetic tail. Journal of Geophysical Research, 88(A9), 6904-6,910. https://doi.org/10.1029/JA088iA09p06904

Desch, M. D., \& Rucker, H. O. (1983). The relationship between Saturn kilometric radiation and the solar wind. Journal of Geophysical Research, 88(A11), 8999-9006. https://doi.org/10.1029/JA088iA11p08999

Desch, M. D., \& Rucker, H. O. (1985). Saturn radio emission and the solar wind: Voyager-2 studies. Advances in Space Research, 5(4), 333-336. https://doi.org/10.1016/0273-1177(85)90159-0

Fuselier, S. A., Frahm, R., Lewis, W. S., Masters, A., Mukherjee, J., Petrinec, S. M., \& Sillanpaa, I. J. (2014). The location of magnetic reconnection at Saturn's magnetopause: A comparison with Earth. Journal of Geophysical Research: Space Physics, 119, 2563-2578 https://doi.org/10.1002/2013JA019684

Gurnett, D. A., Kurth, W. S., Kirchner, D. L., Hospodarsky, G. B., Averkamp, T. F., Zarka, P., et al. (2004). The Cassini radio and plasma wave investigation. Space Science Reviews, 114(1-4), 395-463. https://doi.org/10.1007/s11214-004-1434-0

Huddleston, D. E., Russell, C. T., Le, G., \& Szabo, A. (1997). Magnetopause structure and the role of reconnection at the outer planets. Journal of Geophysical Research, 102(A11), 24,289-24,302. https://doi.org/10.1029/97JA02416

Jackman, C. M., Achilleos, N., Bunce, E. J., Cecconi, B., Clarke, J. T., Cowley, S. W. H., et al. (2005). Interplanetary conditions and magnetospheric dynamics during the Cassini orbit insertion fly-through of Saturn's magnetosphere. Journal of Geophysical Research, 110 (A10), A10212. https://doi.org/10.1029/2005JA011054

Jackman, C. M., Arridge, C. S., Slavin, J. A., Milan, S. E., Lamy, L., Dougherty, M. K., \& Coates, A. J. (2010). In situ observations of the effect of a solar wind compression on Saturn's magnetotail. Journal of Geophysical Research, 115(A10), A10240. https://doi.org/10.1029/ 2010JA015312

Jackman, C. M., Lamy, L., Freeman, M. P., Zarka, P., Cecconi, B., Kurth, W. S., et al. (2009). On the character and distribution of lowerfrequency radio emissions at Saturn, and their relationship to substorm-like events. Journal of Geophysical Research, 114(A8), A08211. https://doi.org/10.1029/2008JA013997

Jackman, C. M., \& Arridge, C. S. (2011). Solar cycle effects on the dynamics of Jupiter's and Saturn's magnetospheres. Solar Phys., 274, 481-502. https://doi.org/10.1007/s11207-011-9748-z

Jackman, C. M., Slavin, J. A., \& Cowley, S. W. H. (2011). Cassini observations of plasmoid structure and dynamics: Implications for the role of magnetic reconnection in magnetospheric circulation at Saturn. Journal of Geophysical Research, 116(A10), A10212. https://doi.org/ $10.1029 / 2011 \mathrm{JA} 016682$

Jackman, C. M., Thomsen, M. F., \& Dougherty, M. K. (2019). Survey of Saturn's magnetopause and bow shock positions over the entire Cassini mission: boundary statistical properties, and exploration of associated upstream conditions. Journal of Geophysical Research: Space Physics. https://doi.org/10.1029/2019JA026628

Jasinski, J. M., Arridge, C. S., Lamy, L., Leisner, J. S., Thomsen, M. F., Mitchell, D. G., et al. (2014). Cusp observation at Saturn's highlatitude magnetosphere by the Cassini spacecraft. Geophysical Research Letters, 41, 1382-1388. https://doi.org/10.1002/ 2014GL059319

Jia, X., Hansen, K. C., Gombosi, T. I., Kivelson, M. G., Tóth, G., DeZeeuw, D. L., \& Ridley, A. J. (2012). Magnetospheric configuration and dynamics of Saturn's magnetosphere: A global MHD simulation. Journal of Geophysical Research, 117(A5), A05225. https://doi.org/ 10.1029/2012JA017575

Kanani, S. J., Arridge, C. S., Jones, G. H., Fazakerley, A. N., McAndrews, H. J., Sergis, N., et al. (2010). A new form of Saturn's magnetopause using a dynamic pressure balance model, based on in situ, multi-instrument Cassini measurements. Journal of Geophysical Research, 115 (A6), A06207. https://doi.org/10.1029/2009JA014262

Kidder, A., Paty, C. S., Winglee, R. M., \& Harnett, E. M. (2012). External triggering of plasmoid development at Saturn. Journal of Geophysical Research, 117(A7), A07206. https://doi.org/10.1029/2012JA017625 
Kimura, T., Lamy, L., Tao, C., Badman, S. V., Kasahara, S., Cecconi, B., et al. (2013). Long-term modulations of Saturn's auroral radio emissions by the solar wind and seasonal variations controlled by the solar ultraviolet flux. Journal of Geophysical Research: Space Physics, 118, 7019-7035. https://doi.org/10.1002/2013JA018833

Krupp, N., Kollmann, P., Mitchell, D. G., Thomsen, M. F., Jia, X., Masters, A., \& Zarka, P. (2018). Global configuration and seasonal variations of Saturn"s magnetosphere. In H. Kevin, F. M. F. Baines, N. Krupp, \& T. Stallard (Eds.), Saturn in the 21st Century, (pp. 126-165). Cambridge, UK: Cambridge University Press. https://doi.org/10.1017/9781316227220.006

Kurth, W. S., Bunce, E. J., Clarke, J. T., Crary, F. J., Grodent, D. C., Ingersoll, A. P., et al. (2009). Auroral processes. In M. K. Dougherty, L. W Esposito, \& S. M. Krimigis (Eds.), Saturn from Cassini-Huygens, (pp. 333-374). New York: Springer Science+Business Media B. V. https://doi.org/10.1007/978-1-4020-9217-6_12

Kurth, W. S., Gurnett, D. A., Clarke, J. T., Zarka, P., Desch, M. D., Kaiser, M. L., et al. (2005). An Earth-like correspondence between Saturn's auroral features and radio emission. Nature, 433(7027), 722-725. https://doi.org/10.1038/nature03334

Kurth, W. S., Hospodarsky, G. B., Gurnett, D. A., Lamy, L., Dougherty, M. K., Nichols, J., et al. (2016). Saturn kilometric radiation intensities during the Saturn auroral campaign of 2013. Icarus, 263, 2-9. https://doi.org/10.1016/j.icarus.2015.01.003

Kurth, W. S., Lecacheux, A., Averkamp, T. F., Groene, J. B., \& Gurnett, D. A. (2007). A Saturnian longitude system based on a variable kilometric radiation period. Geophysical Research Letters, 34, L02201. https://doi.org/10.1029/2006GL028336

Lai, H. R., Wei, H. Y., Russell, C. T., Arridge, C. S., \& Dougherty, M. K. (2012). Reconnection at the magnetopause of Saturn: Perspective from FTE occurrence and magnetosphere size. Journal of Geophysical Research, 117(A5), A05222. https://doi.org/10.1029/2011JA017263

Lamy, L. (2017). The Saturnian kilometric radiation before the Cassini grand finale. In G. Fischer, G. Mann, M. Panchenko, \& P. Zarka (Eds.), Planetary Radio Emissions VIII, edited by, (pp. 171-190). Vienna: Austrian Academy of Sciences Press.

Lamy, L., Prangé, R., Tao, C., Kim, T., Badman, S. V., Zarka, P., et al. (2018). Saturn's northern aurorae at solstice from HST observations coordinated with Cassini's grand finale. Geophysical Research Letters, 45(18), 9353-9362. https://doi.org/10.1029/2018GL078211

Lamy, L., Zarka, P., Cecconi, B., Prangé, R., Kurth, W. S., \& Gurnett, D. A. (2008). Saturn kilometric radiation: Average and statistical properties. Journal of Geophysical Research, 113, A07201. https://doi.org/10.1029/2007JA012900

Lamy, L., Schippers, P., Zarka, P., Cecconi, B., Arridge, C. S., Dougherty, M. K., et al. (2010). Properties of Saturn kilometric radiation measured within its source region. Geophysical Research Letters, 37, L12104. https://doi.org/10.1029/2010GL043415

Lamy, L., Prangé, R., Pryor, W., Gustin, J., Badman, S. V., Melin, H., et al. (2013). Multispectral simultaneous diagnosis of Saturn's aurorae throughout a planetary rotation. Journal of Geophysical Research: Space Physics, 118, 4817-4843. https://doi.org/10.1002/jgra.50404

Masters, A., Eastwood, J. P., Swisdak, M., Thomsen, M. F., Russell, C. T., Sergis, N., et al. (2012). The importance of plasma $\beta$ conditions for magnetic reconnection at Saturn's magnetopause. Geophysical Research Letters, 39, L08103. https://doi.org/10.1029/2012GL051372

Masters, A., Fujimoto, M., Hasegawa, H., Russell, C. T., Coates, A. J., \& Dougherty, M. K. (2014). Can magnetopause reconnection drive Saturn's magnetosphere? Geophysical Research Letters, 41, 1862-1868. https://doi.org/10.1002/2014GL059288

McAndrews, H. J., Owen, C. J., Thomsen, M. F., Lavraud, B., Coates, A. J., Dougherty, M. K., \& Young, D. T. (2008). Evidence for reconnection at Saturn's magnetopause. Journal of Geophysical Research, 113(A4), A04210. https://doi.org/10.1029/2007JA012581

Menietti, J. D., Mutel, R. L., Schippers, P., Ye, S.-Y., Gurnett, D. A., \& Lamy, L. (2011). Analysis of Saturn kilometric radiation near a source center. Journal of Geophysical Research, 116(A12), A12222. https://doi.org/10.1029/2011JA017056

Mitchell, D. G., Brandt, P. C., Roelof, E. C., Dandouras, J., Krimigis, S. M., Mauk, B. H., et al. (2005). Energetic ion acceleration in Saturn's magnetotail: Substorms at Saturn? Geophysical Research Letters, 32, L20S01. https://doi.org/10.1029/2005GL022647

Mitchell, D. G., Krimigis, S. M., Paranicas, C., Brandt, P. C., Carbary, J. F., Roelof, E. C., et al. (2009). Recurrent energization of plasma in the midnight-to-dawn quadrant of Saturn's magnetosphere, and its relationship to auroral UV and radio emissions. Planetary and Space Science, 57, 1732-1742. https://doi.org/10.1016/j.pss.2009.04.002

Park, J.-S., Shue, J.-H., Nariuki, Y., \& Kartalev, M. (2019). Dependence of thermodynamic processes on upstream interplanetary magnetic field conditions for magnetosheath ions. Journal of Geophysical Research: Space Physics, 124(3), 1866-1882. https://doi.org/10.1029/ 2018JA026108

Pilkington, N. M., Achilleos, N., Arridge, C. S., Guio, P., Masters, A., Ray, L. C., et al. (2015). Internally driven large-scale changes in the size of Saturn's magnetosphere. Journal of Geophysical Research: Space Physics, 120, 7289-7306. https://doi.org/10.1002/2015JA021290

Pogorelov, N. V., Borovikov, S. N., Heerikhuisen, J., Tae, K. K., Kryukov, I. A., \& Zank, G. P. (2014). MS-FLUKSS and its application to modeling flows of partially ionized plasma in the heliosphere. In 2014 Annual Conference on Extreme Science and Engineering Discovery Environment ACM Digital Library (New York) Article No. 22. NY, USA: ACM New York. https://doi.org/10.1145/2616498.2616499

Radioti, A., Grodent, D., Gérard, J.-C., Milan, S. E., Bonfond, B., Gustin, J., \& Pryor, W. (2011). Bifurcations of the main auroral ring at Saturn: Ionospheric signatures of consecutive reconnection events at the magnetopause. Journal of Geophysical Research, 116(A11), A11209. https://doi.org/10.1029/2011JA016661

Rathakrishnan, E. (2010). Applied Gas Dynamics. Singapore: John Wiley.

Reed, J. J., Jackman, C. M., Lamy, L., Kurth, W. S., \& Whiter, D. K. (2018). Low-frequency extensions of the Saturn kilometric radiation as a proxy for magnetospheric dynamics. Journal of Geophysical Research: Space Physics, 123(1), 443-463. https://doi.org/10.1002/ 2017JA024499

Roussos, E., Jackman, C. M., Thomsen, M. F., Kurth, W. S., Badman, S. V., Paranicas, C., et al. (2017). Solar energetic particles (SEP) and galactic cosmic rays (GCR) as tracers of solar wind conditions near Saturn: Event lists and applications. Icarus, 300, 47-71. https://doi. org/10.1016/j.icarus.2017.08.040

Schippers, P., Arridge, C. S., Menietti, J. D., Gurnett, D. A., Lamy, L., Cecconi, B., et al. (2011). Auroral electron distributions within and close to the Saturn kilometric radiation source region. Journal of Geophysical Research, 116(A5), A05203. https://doi.org/10.1029/ 2011JA016461

Sergis, N., Jackman, C. M., Masters, A., Krimigis, S. M., Thomsen, M. F., Hamilton, D. C., et al. (2013). Particle and magnetic field properties of the Saturnian magnetosheath: Presence and upstream escape of hot magnetospheric plasma. Journal of Geophysical Research: Space Physics, 118, 1620-1634. https://doi.org/10.1002/jgra.50164

Tao, C., Kataoka, R., Fukunishi, H., Takahashi, Y., \& Yokoyama, T. (2005). Magnetic field variations in the Jovian magnetotail induced by solar wind dynamic pressure enhancements. Journal of Geophysical Research, 110(A11), A11208. https://doi.org/10.1029/ 2004JA010959

Taubenschuss, U., Rucker, H. O., Kurth, W. S., Cecconi, B., Zarka, P., Dougherty, M. K., \& Steinberg, J. T. (2006). Linear prediction studies for the solar wind and Saturn kilometric radiation. Annales de Geophysique, 24(11), 3139-3150. https://www.ann-geophys.net/24/3139/ 2006/, https://doi.org/10.5194/angeo-24-3139-2006

Thomsen, M. F. (2013). Saturn's magnetospheric dynamics. Geophysical Research Letters, 40, 5337-5344. https://doi.org/10.1002/ 2013GL057967 
Thomsen, M. F., Coates, A. J., Jackman, C. M., Sergis, N., Jia, X., \& Hansen, K. C. (2018). Survey of magnetosheath plasma properties at Saturn and inference of upstream flow conditions. Journal of Geophysical Research: Space Physics, 123, 2034-2053. https://doi.org/ $10.1002 / 2018 J A 025214$

Thomsen, M. F., Jackman, C. M., Mitchell, D. G., Hospodarsky, G., Kurth, W. S., \& Hansen, K. C. (2015). Sustained lobe reconnection in Saturn's magnetotail. Journal of Geophysical Research: Space Physics, 120, 10,257-10,274. https://doi.org/10.1002/2015JA021768

Went, D. R., Hospodarsky, G. B., Masters, A., Hansen, K. C., \& Dougherty, M. K. (2011). A new semiempirical model of Saturn's bow shock based on propagated solar wind parameters. Journal of Geophysical Research, 116(A7), A07202. https://doi.org/10.1029/2010JA016349

Zieger, B., \& Hansen, K. C. (2008). Statistical validation of a solar wind propagation model from 1 to 10 AU. Journal of Geophysical Research, 113(A8), A08107. https://doi.org/10.1029/2008JA013046

Zieger, B., Hansen, K. C., Gombosi, T. I., \& De Zeeuw, D. L. (2010). Periodic plasma escape from the mass-loaded Kronian magnetosphere. Journal of Geophysical Research, 115(A8), A08208. https://doi.org/10.1029/2009JA014951 